September 17, 2018

LBNL-41227, UCB-PTH-97/71

hep-th/9805142

\title{
Branes and Electric-Magnetic Duality in Supersymmetric QCD
}

\author{
Kentaro Hori \\ Department of Physics, University of California at Berkeley \\ 366 Le Conte Hall, Berkeley, CA 94720-7300, U.S.A. \\ and \\ Theoretical Physics Group, Mail Stop 50A-5101 \\ Ernest Orlando Lawrence Berkeley National Laboratory \\ Berkeley, CA 94720, U.S.A.
}

\begin{abstract}
We study the Type IIA limit of the $M$ theory fivebrane configuration corresponding to $N=1$ supersymmetric QCD with massless quarks. We identify the effective gauge coupling constant that fits with Novikov-ShifmanVeinshtein-Zakharov exact beta function. We find two different Type IIA limits that correspond to the electric and magnetic descriptions of SQCD, as observed in the massive case by Schmaltz and Sundrum. The analysis is extended to the case of symplectic and orthogonal gauge groups. In any of the cases considered in this paper, the electric and magnetic configurations are smoothly interpolated via $M$ theory. This is in sharp contrast with the proposed derivation of $N=1$ duality within the weakly coupled Type IIA string theory where a singularity is inevitable unless one turns on a parameter that takes the theory away from an interesting point.
\end{abstract}




\section{Introduction}

Supersymmetric gauge theories in various dimensions have been studied by realizing them on the worldvolume of branes and using superstring duality [1]. In particular, the duality between Type IIA string theory and eleven-dimensional supergravity can be used to learn about four-dimensional gauge theories [2-5]. Type IIA brane configuration, on which a gauge theory is realized, is in general singular and hard to analyze, but it becomes smooth and tractable in the eleven-dimensional supergravity limit where the Type IIA string coupling constant $g_{s t}$ becomes large.

The worldvolume theory is coupled to various string excitation modes and depends on parameters such as $g_{s t}$ which have no counterpart in ordinary gauge theory. In particular the theory becomes quantitatively different as we send $g_{s t}$ large, as explicitly shown in [6]. However, some quantities (mainly, BPS or holomorphic objects) are independent of such extra parameters and can be computed by going to a region of the parameter space where the supergravity approximation becomes valid. In addition, as far as there is no singularity in going to such a region, it is likely that qualitative features are unchanged. One remarkable prediction based on this assumption is that the QCD strings can end on domain walls in $N=1$ supersymmetric Yang-Mills theory [4].

In this paper, we show that the two Type IIA configurations of [7] realizing the massless $N=1$ SQCD and the dual magnetic theory [8] are smoothly interpolated by a family of fivebrane configurations in $M$ theory. The smoothness of the interpolation strongly suggests that the universality class of the theory remains the same through the process.

An interpolation of the two configuration was earlier considered within the weakly coupled Type IIA string theory in [7] and in many subsequent papers cited in [1] (see also [9] for a differnt but closely related approach). However, one must turn on a parameter corresponding to the Fayet-Iliopoulos parameter of the $U(1)$ factor of the gauge group in order to avoid a singularity in the process of brane move. It is doubtful whether there is a $U(1)$ factor in the gauge group, but even if we admit the $U(1)$ factor, turning on the FI parameter takes the theory away from an interesting point of the moduli space, and the brane move simply leads to an equivalence of two free or empty theories. One may turn on and off the FI parameter in the course of the brane move, but a similar manipulation, such as turning on and off the mass parameter, will send the theory to the one with less flavors which is definitely different from the original one. Furthermore, in the case of symplectic or orthogonal gauge groups, there is no room for such manipulation $[10,11]$ and the singularity seems unavoidable. 
What is shown in this paper can be considered as the $M$ theory resolution of such a singular brane move in Type IIA string theory. There is no singularity in the process of interpolation even in the case of symplectic and orthogonal gauge groups, and there is no need to change the physical parameters. We also show that the Type IIA brane move with mass parameters turned on and off, as mentioned above, involves a singularity in $M$ theory side and the change of the universality class can be explained.

Interpolating the electric and magnetic configurations via $M$ theory was first discussed in [12] where $N=1 \mathrm{SQCD}$ with massive quarks was considered. Extension to other groups and interactions, again with mass terms for quarks, has also appeared in subsequent works [13-16]. Turning on mass parameter takes the theory away from an interesting point, but the basic idea can be extended to the massless case and will be used in this paper.

This paper is organized as follows.

In section 2, we identify the holomorphic effective gauge coupling at an energy higher than the dynamical scale using the fivebrane configuration whose complex structure is determined in [3]. We show that the wavefunction normalization factor enters in a way compatible with Novikov-Shifman-Veinshtein-Zakharov (NSVZ) exact beta function [21].

In section 3, we consider the Type IIA limit of the $M$ theory fivebrane configuration and show that there are two limits that correspond to the electric and magnetic theories of [8]. We discuss what kind of brane move does not change the universality class of the theory. Some technical details relevant in this section is implicitly given in [3] section 5.4. and are presented more explicitly in [17-19].

In section 4 , we extend the analysis to the case of symplectic gauge group. We show that Type IIA configurations realizing the electric and magnetic theories are smoothly interpolated through a family of fivebrane configurations. We also show that the fivebrane configuration for a dynamical supersymmetry breaking model proposed in [20] has the correct Type IIA limit.

In section 5, we consider the orthogonal groups. We again show that Type IIA configurations realizing the electric and magnetic theories are smoothly interpolated through a family of fivebrane configurations. We uncover a problem in describing exact results in terms of branes for the case $N_{f}=N_{c}-2$ where the theory flows generically to a Maxwell theory.

In an appendix, we correct an error in a field theory argument of [3], and show that the brane prediction on the supersymmetric ground states was correct. 


\section{Branes and Renormalization Group}

In the study of Type IIA brane configurations corresponding to $N=2$ supersymmetric gauge theories in four dimensions [2], the bending of NS5-branes caused by D4-branes ending on them was interpreted as reflecting the running of the gauge coupling constant. In this section, we extend this interpretation to the case of $N=1$ supersymmetric QCD, by considering a suitable Type IIA limit of the configuration of $M$ theory fivebrane constructed in [3]. We will see that the wavefunction normalization factor enters correctly in the formula for the effective gauge coupling constant, which is relevant for the derivation of NSVZ beta function of [21].

\section{$2.1 \quad$ A Simple Example}

We start with reviewing the $M$ theory fivebrane configuration for $N=2 S U\left(N_{c}\right)$ superYang-Mills theory. The configuration is in the flat eleven-dimensional spacetime where the eleventh direction is compactified on a circle of radius $R$. We introduce the time and space cooredinates $x^{0}, x^{1}, \ldots, x^{10}$ with respect to which the flat metric is expressed as

$$
\mathrm{d} s^{2}=-\left(\mathrm{d} x^{0}\right)^{2}+\left(\mathrm{d} x^{1}\right)^{2}+\cdots+\left(\mathrm{d} x^{9}\right)^{2}+R^{2}\left(\mathrm{~d} x^{10}\right)^{2},
$$

where $x^{10}$ parametrize the compact eleven-th direction, $x^{10} \equiv x^{10}+2 \pi$. The worldvolume

of the fivebrane spans the four-dimensional space-time in the $x^{0,1,2,3}$ directions and wraps on a two-dimensional surface embedded in the four-manifold $\mathbf{R}^{3} \times S^{1}$ at $x^{7}=x^{8}=x^{9}=0$ parametrized by $x^{4,5,6,10}$. We give a complex structure to $\mathbf{R}^{3} \times S^{1}$ by a choice of complex coordinates

$$
\begin{aligned}
& v=\ell_{s t}^{-2}\left(x^{4}+i x^{5}\right), \\
& t=\exp \left(-\frac{x^{6}}{R}-i x^{10}\right) .
\end{aligned}
$$

In (2.2) we introduced the prefactor $\ell_{s t}{ }^{-2}$, the tension of Type IIA strings, so that $|\Delta v|$ measures the mass of an open string stretched between an interval $\Delta v$.

The supersymmetric configuration for $N=2 S U\left(N_{c}\right)$ super-Yang-Mills theory is given by the fivebrane wrapped on the Riemann surface in the $t-v$ space described by

$$
t+t^{-1}=\frac{1}{\Lambda^{N_{c}}} \prod_{a=1}^{N_{c}}\left(v-\phi_{a}\right),
$$

where $\Lambda$ is a parameter that will be identified with the dynamical scale of the superYang-Mills theory. The parameters $\phi_{a}$ are interpreted as the eigenvalues of the vev of the adjoint chiral superfield. 
For the values of $R$ and $\Lambda$ small compared to some length $L$ and energy $E$, the Riemann surface (2.4) looks like two flat sheets connected by thin necks, with respect to the scales set by $L$ and $E$ in the $x^{6}$ and $v$ directions. The distiction between the regions of the flat sheets and thin necks becomes sharper as $L / R$ and $E / \Lambda$ becomes larger. If we send these quantities very large by keepng the combination $\mathrm{e}^{L / 2 R}(\Lambda / E)^{N_{c}}$ to be finite, say 1 , the sheet regions and the neck region are separated at $x^{6}= \pm L / 2$. See Figure 1 in which we plot $|v|$ as a function of $x^{6}$ (for a fixed $x^{10}$ ) in the case of $N_{c}=2$ where we set $L=E=1$ and we put $\phi_{a}=0$.
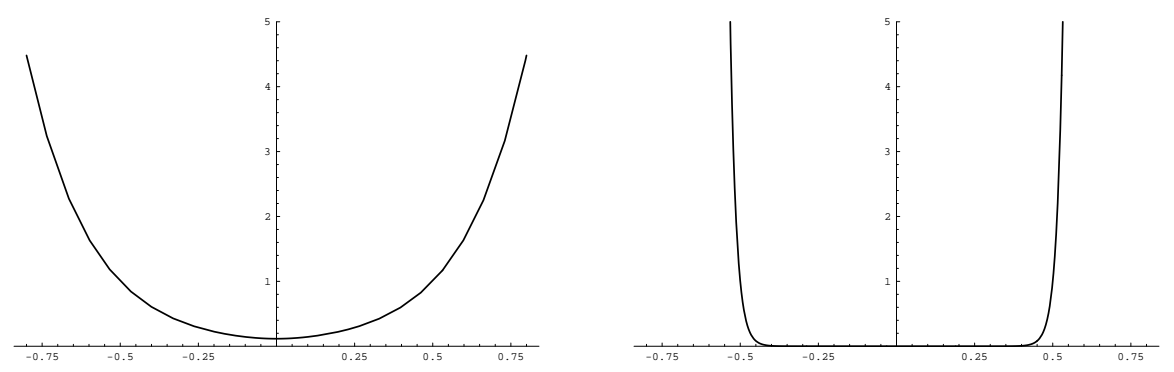

Figure 1: Graphs of $|v|=\sqrt{\mathrm{e}^{-\frac{0.5}{R}}\left(\mathrm{e}^{\frac{x^{6}}{R}}+\mathrm{e}^{-\frac{x^{6}}{R}}\right)}$ for $R=0.1$ (left) and $R=0.01$ (right)

In the weakly coupled Type IIA limit $R \ll \ell_{11}$, the flat sheets and thin necks correspond to the NS5-branes and D4-branes respectively. Then, $L$ is interpreted as the length of the D4-branes stretched in the $x^{6}$ direction between the two NS5-branes. Since $1 / R=1 /\left(g_{s t} \ell_{s t}\right)$ is the inverse square of the gauge coupling constant of the $4+1$ dimensional theory of the D4-brane worldvolume, $L / R$ is identified as the inverse square of the gauge coupling constant of the four-dimensional theory obtained by compactification on the interval of length $L$ :

$$
\frac{L}{R}=\frac{1}{g^{2}} .
$$

As we change $E$ with $\Lambda$ being fixed, the ratio $L / R$ changes according to

$$
\mathrm{e}^{-L / R}=\left(\frac{\Lambda}{E}\right)^{2 N_{c}}
$$

In [2], this was interpreted as reflecting the running of the gauge coupling constant.

Actually, $g^{2}$ in (2.5) can be indentified as the effective gauge coupling constant at the energy scale $E$ of the asymptotic free $S U\left(N_{c}\right)$ Yang-Mills theory. In particular, (2.6) can be used to identify the parameter $\Lambda$ as the dynamical scale of the theory. This follows from the following reasoning. Consider for instance two D4-branes sepatated by a 
distance $\Delta \ell$ which is smaller than the string length $\ell_{s t}$. At energies below the string scale $M_{s t}=1 / \ell_{s t}$, the theory on the worldvolume is the $U(2)$ super-Yang-Mills theory. The non-abelian gauge symmetry is unbroken at energies above the W-bosin mass $\ell_{s t}{ }^{-2} \Delta \ell$, but is broken to the abelian subgroup below this scale. In other words, at a fixed energy scale $E$ below $M_{s t}$, the $S U(2)$ gauge symmetry is unbroken if the separation is small enough $\Delta \ell<\ell_{s t}{ }^{2} E$. Let us go back to our configuration. At large values of $\left|x^{6}\right|$ such that $v$ is much larger than $\Lambda$ and $\phi_{a}$ 's, the curve is roughly described by $v=\Lambda \mathrm{e}^{\left|x^{6}\right| / N_{c} R \pm i x^{10} / N_{c}}$. In the interval $-L / 2<x^{6}<L / 2$, the configuration looks as the $N_{c}$ D4-branes which are nearly on top of each other compared to the length scale $\ell_{s t}{ }^{2} E$, since the value of $v$ is $E$ at $x^{6}= \pm L / 2$ and exponentially small as $\left|x^{6}\right|$ descreases. Thus, the $S U\left(N_{c}\right)$ gauge symmetry is unbroken at the energy $E$ in this interval. Outside the interval, the separation of the D4-branes is much greater than $\ell_{s t}{ }^{2} E$ and the W-bosons decouple at an energy above $E$. Or more precisely, we see that the picture of aligned D4-branes is actually incorrect in this region and there is no obvious candidate for the W-bosons. In any case, we do not have any non-trivial gauge dynamics at the energy $E$ from the region outside the interval $-L / 2<x^{6}<L / 2$. Therefore we can consider $g$ given by (2.5) as the gauge coupling constant set at the energy $E$ as far as $E<1 / L$, and hence we can identify the parameter $\Lambda$ as the dynamical scale of the four-dimensional gauge theory.

The holomorphic description of the fivebrane is of course valid in the region of the parameter space where all the characteristic lengths of the brane and space-time are much larger than the eleven-dimensional Planck length, in particular $R \gg \ell_{11} \gg \ell_{s t}$ and $\Lambda \gg \ell_{s t}^{-2} \ell_{11} \gg M_{s t}$. Since this is not the region where the gauge dynamics is dominant, one may wonder whether the measurement of the gauge coupling based on the holomorphic description is valid. The point is that we can scale up the lengths $R, L$ and energies $\Lambda, E$ to the region where holomorphic description is valid while keeping the ratio $L / R$ and $\Lambda / E$ fixed. Then, the gauge coupling constant measured as (2.5)-(2.6) is invariant under the rescaling, and therefore it can be considered to be valid even when we scale down the parameters back to the gauge theory region.

\section{2 $N=1$ Supersymmetric QCD}

We next consider a configuration of the fivebrane corresponding to $N=1$ SQCD with gauge group $S U\left(N_{c}\right)$ and $N_{f} \geq N_{c}$ flavors. The complex structure of the configuration was determined in [3] as we will revisit shortly.

The fivebrane configuration is in the eleven-dimensional space-time which is a product of the flat seven-dimensional space-time (in the $x^{0,1,2,3,7,8,9}$ directions) and the Taub-NUT 
space of $A_{N_{f}-1}$ type (in the $x^{4,5,6,10}$ directions). Thus, the metric of the eleven-dimensional space-time is given by

$$
\mathrm{d} s^{2}=-\left(\mathrm{d} x^{0}\right)^{2}+\left(\mathrm{d} x^{1}\right)^{2}+\left(\mathrm{d} x^{2}\right)^{2}+\left(\mathrm{d} x^{3}\right)^{2}+\mathrm{d} s_{\mathrm{TN}}^{2}+\left(\mathrm{d} x^{7}\right)^{2}+\left(\mathrm{d} x^{8}\right)^{2}+\left(\mathrm{d} x^{9}\right)^{2}
$$

where $\mathrm{d} s_{\mathrm{TN}}^{2}$ is the Taub-NUT metric which will be given below. The fivebrane spans the $x^{0,1,2,3}$ directions and wraps on a Riemann surface in the transversal directions.

The Taub-NUT geometry in $M$ theory corresponds to D6-branes in Type IIA string theory which can be used to realize the quark multiplets. If the D6 branes are at $\vec{x}=$ $\left(x^{4}, x^{5}, x^{6}\right)=\vec{x}_{i}, i=1, \ldots, N_{f}$, the Taub-NUT metric is given by

$$
\mathrm{d} s_{\mathrm{TN}}^{2}=U \mathrm{~d} \vec{x}^{2}+R^{2} U^{-1}\left(\mathrm{~d} x^{10}+\vec{\omega} \cdot \mathrm{d} \vec{x}\right)^{2},
$$

where

$$
U=1+\sum_{i=1}^{N_{f}} \frac{R}{2\left|\vec{x}-\vec{x}_{i}\right|}, \quad \operatorname{curl} \vec{\omega}=\operatorname{grad} \sum_{i=1}^{N_{f}} \frac{1}{\left|\vec{x}-\vec{x}_{i}\right|} .
$$

Since $x^{10}$ is a periodic coordinete of period $2 \pi$, the Taub-NUT space can be considered as a circle bundle over the $\vec{x}$ space where the radius $R / \sqrt{U}$ shrinks at the position of the sixbranes. At large $|\vec{x}|$, the asymptotic radius is $R$ and the equation (2.9) shows that the circle bundle is the monopole bundle of magnetic charge $N_{f}$. In what follows, we place the sixbranes at $N_{f}$ points in the $x^{6}$-axis $x_{i}^{4}=x_{i}^{5}=0$ so that the configuration we will consider corresponds to massless SQCD.

\section{Holomorphic Description of the Configuration}

In [3] we have given a holomorphic description of the fivebrane configuration which we now review.

We consider the space transverse to the four dimensions $x^{0,1,2,3}$ to be the product of a real line (the $x^{7}$ direction) and a three-dimensional complex manifold (product of the Taub-NUT space and the $x^{8,9}$ plane). We select one of the complex structures of the Taub-NUT space such that

$$
v=\text { const } \times\left(x^{4}+i x^{5}\right)
$$

is a complex coordinate. We also provide the $x^{8,9}$ plane with a complex coordinate

$$
w=\text { const } \times\left(x^{8}+i x^{9}\right) .
$$

Under the choice (2.10) of the complex structure, other complex coordinates of the TaubNUT space can be given by

$$
y=c \times \mathrm{e}^{-\left(x^{6} / R+i x^{10}\right)} \prod_{i=1}^{N_{f}} \sqrt{\left|\vec{x}-\vec{x}_{i}\right|-\left(x^{6}-x_{i}^{6}\right)},
$$




$$
x=c^{-1} \times\left(\frac{v}{\left|x^{4}+i x^{5}\right|}\right)^{N_{f}} \mathrm{e}^{x^{6} / R+i x^{10}} \prod_{i=1}^{N_{f}} \sqrt{\left|\vec{x}-\vec{x}_{i}\right|+\left(x^{6}-x_{i}^{6}\right)},
$$

where $c$ is a constant. It is a matter of convention that the proportionality constant in (2.13) is chosen to be inverse to $c$ in (2.12). The complex coordinates $y, x$ and $v$ are related by

$$
y x=v^{N_{f}} .
$$

It appears from this that the Taub-NUT space has a singularity at $y=x=v=0$ which is indeed the singularity of $A_{N_{f}-1}$ type when the sixbranes are on top of each other. However, when the D6-branes are separated in the $x^{6}$ direction, the singular point $y=x=v=0$ is blown up to $N_{f}-1 \mathrm{CP}^{1}$ cycles and the singularity is resolved as we can see by introducing an appropriate coordinate system around each of the D6-branes (see the next section).

The fivebrane is wrapping a holomorphic curve consisting of two infinite components and several $\mathrm{CP}^{1}$ components. The infinite components are described by

$$
C^{\prime}\left\{\begin{array} { l } 
{ y = \prod _ { i = 1 } ^ { N _ { c } } ( w - M _ { i } ) } \\
{ v = 0 }
\end{array} \quad C \left\{\begin{array}{l}
x=\Lambda^{-\left(3 N_{c}-N_{f}\right)} v^{N_{c}} \\
w=0
\end{array}\right.\right.
$$

while the $\mathrm{CP}^{1}$ components at $y=x=v=0$ will be described in the next section.

This was obtained in [3] by rotation and deformation of the configuration corresponding to the $N=2$ SQCD. ( $\widetilde{y}$ in $[3]$ is denoted simply by $y$ here and the normalization of $x$ is also changed.) The parameter $\Lambda$ in the above expression was identified in [3] as the dynamical scale of the $N=1$ SQCD by imposing the renormalization group matching with the high energy $N=2$ theory. In this paper, we will see this directly by identifying the gauge coupling constant. The parameters $M_{i}$ are interpreted as the eigenvalues of the vacuum expectation value of the meson matrix $M$. The fact that the number of $M_{i}$ 's is $N_{c}$ reflects the classical (= quantum) constraint $\operatorname{rank} M \leq N_{c}$ on the meson matrix. In addition, by identifying the parameters corresponding to the baryon vevs, we can derive in the brane description the quantum modified constraint for the theory with $N_{f}=N_{c}$ as well as the classical (= quantum) constraint for higher $N_{f}>N_{c}$ concerning the relation between meson and baryon vevs [3]. ${ }^{1}$

\footnotetext{
${ }^{1}$ In [3], there was a gap between the brane and field theory analysis on the quantum constraint for the system with a heavy adjoint chiral multiplet. In Appendix, we will show that this gap is because of our error in the field theory analysis and that the brane prediction is correct.
} 


\section{Extra Parameters}

The description of the holomorphic curve given above does not completely specify the fivebrane configuration in the eleven-dimensional space-time since we have left undetermined the proportionality constants in (2.10)-(2.12).

These constants are constrained if we require that the brane must correctly reproduce the superpotential of the gauge theory. In [4] the superpotential of the worldvolume theory was defined as a certain integral of the holomorphic three-form of the space-time CalabiYau three-fold in which the curve is embedded. ${ }^{2}$ In the present case, the three-fold is the product of the Taub-NUT space and the $x^{8,9}$ plane with the complex structure chosen above, and the three-form is $\mathrm{d}\left(x^{4}+i x^{5}\right) \wedge \mathrm{d}\left(x^{8}+i x^{9}\right) \wedge \mathrm{d}\left(x^{6}+i R x^{10}\right)$ at large $\left|x^{4,5,6}\right|$. As has been shown in appendix A of [6] in the example of $N=1$ super-Yang-Mills theory, in order for the brane computation to reproduce the field theory result, the holomorphic three-form multiplied by the fivebrane tension $\ell_{11}{ }^{-6}$ must be given by

$$
\Omega=\mathrm{d} v \wedge \mathrm{d} w \wedge \frac{\mathrm{d} y}{y} .
$$

This requires the product of the constants in (2.10) and (2.11) to be equal to $R \ell_{11}{ }^{-6}$. Then, the coordinates $v$ and $w$ can be expressed as

$$
v=\ell_{s t}{ }^{-2} Z\left(x^{4}+i x^{5}\right), \quad w=\ell_{11}{ }^{-3} Z^{-1}\left(x^{8}+i x^{9}\right),
$$

where $Z$ is a constant.

Actually, there is a redundancy in the parametrization. Obviously, a phase shift of the constant $c$ in (2.12)-(2.13) can be compensated by a shift of the origin of $x^{10}$. Also, a change of the magnitude of $c$ can be absorbed by an overall shift of $x_{i}^{6}$ 's, and only the combination $c \mathrm{e}^{-\sum_{i} x_{i}^{6} / R}$ characterizing the relative separation of the fivebrane system from the sixbranes in the $x^{6}$ direction has an invariant meaning. Furthermore, the configuration is invariant under the change of parameters as

$$
\begin{aligned}
& Z \rightarrow \zeta^{-1} Z, \quad c \rightarrow \zeta^{N_{c}} c, \\
& M_{i} \rightarrow \zeta M_{i}, \\
& \Lambda^{3 N_{c}-N_{f}} \rightarrow \zeta^{N_{f}} \Lambda^{3 N_{c}-N_{f}}
\end{aligned}
$$

as it can be compensated by the coordinate transformation $v \rightarrow \zeta^{-1} v, w \rightarrow \zeta w, y \rightarrow$ $\zeta^{N_{c}} y$ and $x \rightarrow \zeta^{-N_{c}-N_{f}} x$. This transformation can be identified with the action of the

\footnotetext{
${ }^{2}$ The holomorphic three-form of a Calabi-Yau three-fold is a nowhere vanishing holomorphic threeform such that its wedge product with its complex conjugate is the same as the cube of the Kähler form.
} 
complexified axial $U(1)$ flavor symmetry group which is anomalously broken to $\mathbf{Z}_{N_{f}}$. The radial part is the group of rescalings of the quark field as can be seen in (2.19), and the transformation of $\Lambda$ in (2.20) reflects its anomaly [23] (see also [24]) as we will see after identifying the gauge coupling constant.

We conclude that the configuration depends on two real parameters, $Z^{N_{c}} c \mathrm{e}^{-\sum_{i} x_{i}^{6} / R}$ and $R$, as well as the separation $x_{i}^{6}-x_{i+1}^{6}$ of the D6-branes, in addition to the parameter and observables of the four-dimensional gauge theory $\left(\Lambda, M_{i}\right.$ 's and the location and the chiral two-form of the $\mathrm{CP}^{1}$ components)

\section{The Effective Gauge Coupling Constant}

As in the case of $N=2$ super-Yang-Mills theory, we want to distinguish the region of the fivebrane where the gauge dynamics is relevant at an energy $E$ below the string scale $1 / \ell_{s t}$, by setting an appropriate length scale corresponding to $E$. In the present case, the fivebrane is extending in two directions, $v$ and $w$, and we must set a scale for each of them. We propose that these scales, $v(E)$ and $w(E)$, are related by $v(E) w(E)=E^{3}$. This is based on the fact that the holomorphic three-form $\Omega=\mathrm{d} v \wedge \mathrm{d} w \wedge \mathrm{d} y / y$ should transform as $\Omega \rightarrow \lambda^{3} \Omega$ under a change of the energy scale $E \rightarrow \lambda E$ since the superpotential is given by an integral of $\Omega$ and has mass dimension three.

Let us first determine the scale in the $v$ direction. Note that only the component $C$ is extending in this direction. Since this part of the fivebrane is the same as a part of the $N=2$ configuration, as far as the effect of the other part of the fivebrane is negligible, we can say that the gauge dynamics is relevant in the region where the configuration looks as nearly coincident D4-branes compared to the length scale $\ell_{s t}{ }^{2} E$ just as in the $N=2$ theory. Since the coordinates $x^{4,5}$ and $v$ are related by $(2.17)$, this region is given by $|v|<Z E$. Note that the value of $y$ at the boundary $v=Z E$ is

$$
y(E)=\Lambda^{3 N_{c}-N_{f}}(Z E)^{N_{f}-N_{c}}
$$

Since the scale in the $v$-direction is set by $v(E)=Z E$, the scale in the $w$ direction is set by $w(E)=Z^{-1} E^{2}$. This corresponds to a length scale $\ell_{11}{ }^{3} Z w \sim \ell_{s t}{ }^{2} R E^{2}$ which is much shorter than $\ell_{s t}{ }^{2} E$ in the weakly coupled Type IIA limit $E<1 / \ell_{s t} \ll 1 / R$. Actually, we can provide the following interpretation of this new scale $w(E) \propto E^{2}$. The $N_{c} w$-values of the component $C^{\prime}$ for a fixed $y$ can be interpreted as the non-zero eigenvalues of the meson matrix $\widetilde{Q} Q$ at $y$, as these values at $y=0$ are interpreted as their vacuum expectation values. Since non-zero values of $Q, \widetilde{Q}$ give a mass of order $\sqrt{\widetilde{Q} Q}$ to the gluons, the gauge symmetry is unbroken at the energy $E$ in the region where $\sqrt{w}$ is smaller than a value 
of order $E$. The precise value $Z w=E^{2}$ suggests that the quark kinetic term is given by $Z\left(Q^{\dagger} Q+\widetilde{Q} \widetilde{Q}^{\dagger}\right)$ and the mass of the gauge boson is $\sqrt{Z \widetilde{Q} Q}$. Thus, the gauge dynamics is important at the energy $E$ only in the region $\sqrt{|Z w|}<E$, or $|Z w|<E^{2}$, where $|y|$ is smaller than

$$
y^{\prime}(E)=\left(Z^{-1} E^{2}\right)^{N_{c}} .
$$

To summarize, the $S U\left(N_{c}\right)$ gauge dynamics is relevant in the region $|v|<Z E$ and $|w|<$ $Z^{-1} E^{2}$, and the value of $y$ at the boundaries are $y(E)$ and $y^{\prime}(E)$ given by $(2.21)$ and $(2.22)$ respectively.

We now want to measure the effective gauge coupling constant $g(E)$ at the energy $E$. In the case of $N=2$ super-Yang-Mills theory, we have seen that $1 / g^{2}(E)$ is $1 / R$ times the length in the $x^{6}$-direction of the part of the fivebrane in which the gauge dynamics is relevant at the energy $E$. In other words, $\mathrm{e}^{-1 / g^{2}(E)}$ is given by the ratio of the values of the coordinate $t=\mathrm{e}^{-x^{6} / R-i x^{10}}$ at the two boundaries of the relevant part. The holomorphic coordinate analogous to $t$ is $y$ in the present case: It is the only complex coordinate that has an asymptotic behaviour $\sim \mathrm{e}^{-x^{6} / R-i x^{10}}$ at large $x^{6}$ and does not vanish at a generic point in both of the components $C$ and $C^{\prime}$. Therefore, we propose that $\mathrm{e}^{-1 / g^{2}(E)}$ is likewise given by the ratio of the values of $y$ at the boundaries of the relevant region, i.e. $y(E) / y^{\prime}(E)$.

This may require some explanation. The logarithm of the ratio of the two values of $y$ is in general not close to $1 / R$ times the distance of the boundaries. This is not a problem since the definition of the gauge coupling constant is likely to be corrected by the intersection with the sixbranes. At present, we do not know the precise way to measure the gauge coupling constant in such a situation. Fortunately, there is one parameter $c$ corresponding to the relative position of the sixbranes and the fivebrane system in the $x^{6}$ direction, which has no counterpart in the four-dimensional gauge theory. It is expected that the change of $c$ is irrelevant in gauge theory as far as the curve is smoothly deformed. Especially, a change of $c$ does not affect the holomorphic object, such as holomorphic gauge coupling constant. We can tune $c$ so that all the sixbranes are sent far to the right $\left(x_{i}^{6}\right.$ large). Then, the sixbranes goes away from the region sandwiched by the two boundaries. In the limit $x_{i}^{6} \rightarrow+\infty$ where the effect of the intersection with the sixbranes is suppressed, $\left|\log \left(y(E) / y^{\prime}(E)\right)\right|$ approaches $1 / R$ times the actual distance of the two boundaries.

We thus obtain

$$
\mathrm{e}^{-1 / g^{2}(E)}=\frac{y(E)}{y^{\prime}(E)}=\left(\frac{\Lambda}{E}\right)^{3 N_{c}-N_{f}} Z^{N_{f}} .
$$

This is the same as the one-loop running of the gauge coupling constant of the SQCD. The appearance of the factor $Z^{N_{f}}$ is actually consistent with the interpretation of $Z$ as the 
normalization factor of the quark kinetic term $Z\left(Q^{\dagger} Q+\widetilde{Q} \widetilde{Q}^{\dagger}\right)$ with respect to the fields $Q, \widetilde{Q}$ such that the meson matrix $\widetilde{Q} Q$ is identified with the parameters $M_{i}$. In order to show this, we first note that the gauge coupling in $N=1$ SQCD depends on the choice of variables for the quark multiplet. Rescaling of the quark superfields $Q \rightarrow \sqrt{\zeta} Q, \widetilde{Q} \rightarrow \sqrt{\zeta} \widetilde{Q}$ generates a Jacobian which shifts the inverse square of the gauge coupling constant as $\mathrm{e}^{-1 / g^{2}} \rightarrow \zeta^{N_{f}} \mathrm{e}^{-1 / g^{2}}[23,24]$. The standard and unambiguous prescription to define the gauge coupling constant is to choose the variable such that the quark kinetic term is canonically normalized at a given energy $E$. Since we have made no choice of variables when defining the coupling constant $g(E)$ as $(2.23)$, it is natural to consider it as gauge coupling constant defined in the standard way. By using the rescaling symmetry (2.18) with $\zeta=Z$, we can make $Z \rightarrow 1$ and this induces the change of parameters as $M_{i} \rightarrow Z M_{i}$ and $\Lambda^{3 N_{c}-N_{f}} \rightarrow Z^{N_{f}} \Lambda^{3 N_{c}-N_{f}}$ (2.19)-(2.20). With respect to the new variables $M_{i}^{\prime}=Z M_{i}$ and $\Lambda^{\prime 3 N_{c}-N_{f}}=Z^{N_{f}} \Lambda^{3 N_{c}-N_{f}}$, the quark kinetic term is canonically normalized and the gauge coupling constant $g(E)$ has the standard expression $\mathrm{e}^{-1 / g^{2}(E)}=\left(\Lambda^{\prime} / E\right)^{3 N_{c}-N_{f}}$. The gauge coupling constant $\hat{g}(E)$ defined with respect to the old variables $M_{i}$ is given by $\mathrm{e}^{-1 / \hat{g}^{2}(E)}=(\Lambda / E)^{3 N_{c}-N_{f}}$ and the relation to the standard one (2.23) reflects the rescaling anomaly of $[23]$.

In supersymmetric field theory, the gauge coupling constant receives essentially only the one-loop correction, and (2.23) describes the exact renormalization group flow provided $Z$ changes according to the wavefunction renormalization $Z \rightarrow Z^{\prime}$ under a change of the energy scale $E \rightarrow E^{\prime}[21]$ (see also [24]). In the brane picture, $Z$ is a fixed parameter and hence it appears that the brane does not capture the effect of the wavefunction renormalization. However, the renormalization $Z \rightarrow Z^{\prime}$ of the kinetic term of $Q, \widetilde{Q}$ suggests that the scale in the $w$ direction must change from $Z w(E)=E^{2}$ to $Z^{\prime} w\left(E^{\prime}\right)=$ $E^{\prime 2}$. Then, the scale in the $v$ direction changes as $v\left(E^{\prime}\right)=Z^{\prime} E^{\prime}$, and the gauge coupling constant at the energy $E^{\prime}$ is given by $\mathrm{e}^{-1 / g^{2}\left(E^{\prime}\right)}=\left(\Lambda / E^{\prime}\right)^{3 N_{c}-N_{f}} Z^{\prime N_{f}}$. The flow $g(E) \rightarrow$ $g\left(E^{\prime}\right)$ is nothing but what arizes from the exact beta function of [21]. (To be precise, the beta function of [21] is concerned with "1PI" coupling whereas what we have been considering is the holomorphic, or "Wilsonian" coupling. The relation between them is known [22], and translates the flow described above to the flow of "1PI" coupling of [21].) Note that the length scale in the $v$-direction has changed as $\ell_{s t}{ }^{2} E \rightarrow \ell_{s t}{ }^{2} \frac{Z^{\prime}}{Z} E^{\prime}$. This may be interpreted as the effect on the component $C$ from other components of the fivebrane, which we can no longer neglect as $E$ is reduced since the boundary becomes closer to the other component $C^{\prime}$. 
$N=2$ Broken to $N=1$

In order to examine the above idea to identify the gauge coupling constant, we consider $N=2$ SQCD with $N=2$ broken to $N=1$ by giving a mass $\mu_{\text {a }}$ to the adjoint chiral multiplet. This model has been analyzed in [3] in the brane picture and it was shown that there are various barnches of the moduli space.

When we only look at high energies compared to the scales determined by the vevs or the dynamical scale there is no difference between the various branches, and therefore we only look at the simplest one, the baryonic branch. The curve of the baryonic branch consists of several $\mathrm{CP}^{1}$ components and the two infinite components given by

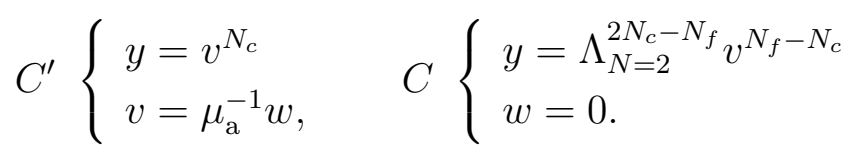

At an energy $E$, the scale in the $v$ and $w$ directions are set by $v(E)=E$ and $w(E)=E^{2}$ (here we set $Z=1$ ignoring the wavefunction renormalization for simplicity).

The component $C$ extends only in the $v$ direction, and the gauge dynamics is relevant at $E$ in the region with $|v|<E$. The value of $y$ at the boundary is $y(E)=\Lambda_{N=2}^{2 N_{c}-N_{f}} E^{N_{f}-N_{c}}$.

On the other hand, the component $C^{\prime}$ extends in the mixed direction $w=\mu_{\mathrm{a}} v$, and the region where the gauge dynamics is relevant depends on the value of $E$ compared to $\mu_{\mathrm{a}}$. For the values of $v$ above $v(E)=E$ the gauge group is broken by the adjoint Higgs field whereas $w \sim E^{2}$ is the scale where the gauge group is broken by the quark field. Note that the value of $v$ at the quark Higgsing scale is $v_{\mathrm{H}}(E)=\mu_{\mathrm{a}}^{-1} E^{2}$ in the component $C^{\prime}$.

- When the energy $E$ is much larger than $\mu_{\text {a }}$, the adjoint Higgsing scale is smaller $v(E) \ll$ $v_{\mathrm{H}}(E)$ and hence the relevant region is $|v|<|v(E)|$ with the boundary value of $y$ being $y^{\prime}(E)=E^{N_{c}}$. Then, the gauge coupling constant is given by

$$
\mathrm{e}^{-1 / g^{2}(E)}=\frac{y(E)}{y^{\prime}(E)}=\frac{\Lambda_{N=2}^{2 N_{c}-N_{f}}}{E^{2 N_{c}-N_{f}}}
$$

and runs in the same way as in the original $N=2$ SQCD.

- When the energy $E$ is much smaller than $\mu_{\mathrm{a}}$, quark Higgsing scale is smaller $v_{\mathrm{H}}(E) \ll$ $v(E)$, and hence the relevant region is $|v|<\left|v_{\mathrm{H}}(E)\right|$ with the boundary value of $y$ being $y^{\prime}(E)=\left(\mu_{\mathrm{a}}^{-1} E^{2}\right)^{N_{c}}$. Thus, the gauge coupling constant is given by

$$
\mathrm{e}^{-1 / g^{2}(E)}=\frac{y(E)}{y^{\prime}(E)}=\frac{\mu_{\mathrm{a}}^{N_{c}} \Lambda_{N=2}^{2 N_{c}-N_{f}}}{E^{3 N_{c}-N_{f}}}
$$


and runs in the same way as in $N=1 \mathrm{SQCD}$ with the dynamical scale $\Lambda$ given by $\Lambda^{3 N_{c}-N_{f}}=\mu_{\mathrm{a}}^{N_{c}} \Lambda_{N=2}^{2 N_{c}-N_{f}}$.

This agrees with the behaviour of the effective gauge coupling in field theory.

\section{Electric-Magnetic Duality}

In this section, we consider the Type IIA limit of the configuration for $N=1 \mathrm{SQCD}$ in more detail. Following the idea of [12], we will find two different limits which coincide with the Type IIA configurations of [7] corresponding to the electric and magnetic description of the theory. The two limits are smoothly interpolated, and hence we may expect the universality class of the theory to be unchanged through the process.

\section{Sixbranes and $\mathrm{CP}^{1}$ Cycles}

As mentioned in the previous section, the description of the Taub-NUT space in terms of the coordinates $y, x, v$ breaks down near the locus of the sixbranes. As far as the sixbranes are separated (in the $x^{6}$ direction in the present case), the Taub-NUT space is smooth and is described by introducing one coordinate system $\left(y_{i}, x_{i}\right)$ in a neighborhood $U_{i}$ of each of the D6-branes $\left(i=1, \ldots, N_{f}\right)$. These coordinates are related to $y, x, v$ by

$$
y=y_{i}^{i} x_{i}^{i-1}, \quad x=y_{i}^{N_{f}-i} x_{i}^{N_{f}+1-i} \text {, and } v=y_{i} x_{i} .
$$

and are related to each other in the overlapping region $U_{i} \cap U_{i+1}$ by

$$
x_{i} y_{i+1}=1 \text { and } y_{i} x_{i}=y_{i+1} x_{i+1} .
$$

There are $N_{f}-1 \mathrm{CP}^{1}$ cycles $C_{1}, \ldots, C_{N_{f}-1}$. The cycle $C_{i}$ is defined as the locus of $y_{i}=0$ in $U_{i}$ and $x_{i+1}=0$ in $U_{i+1}$. Or equivalently, by considering the Taub-NUT space as the circle bundle over the $x^{4,5,6}$ space, $C_{i}$ can also be defined as the fibres over the straight segment in the $x^{6}$ axis stretched between the $i$-th and the $(i+1)$-th D6-branes. In particular, $C_{i-1}$ and $C_{i}$ intersect transversely at the position of the $i$-th D6-brane. The volume of the cycle $C_{i}$ is $\left|x_{i}^{6}-x_{i+1}^{6}\right| R$.

In the weak coupling Type IIA limit $R \ll \ell_{s t}$, the $\mathrm{CP}^{1}$ cycle $C_{i}$ looks like a thin cigar connecting the two D6-branes. A fivebrane wrapped on $C_{i}$ becomes a D4-brane stretched beteen the $i$-th and $(i+1)$-th D6-branes. Likewise, a membrane wrapped on $C_{i}$ becomes an open string stretched beteen these D6-branes and has a mass of order $\ell_{11}{ }^{-3}\left|x_{i}^{6}-x_{i+1}^{6}\right| R=\ell_{s t}{ }^{-2}\left|x_{i}^{6}-x_{i+1}^{6}\right|$. Such membranes can be identified as the $\mathrm{W}$-bosons on 
the worldvolume of the D6-branes. In the limit where all the D6-branes become on top of each other, there is an enhanced $S U\left(N_{f}\right)$ gauge symmetry on the D6-brane worldvolume which is a flavor symmetry from the point of view of the worldvolume theory on the fivebrane. Since this is present already for $N=2$ configurations, it corresponds to the diagonal subgroup of the chiral flavor symmetry group of the SQCD.

\section{$\mathrm{CP}^{1}$ Components of the Fivebrane}

In addition to the two infinite components $C$ and $C^{\prime}$ of (2.15), the fivebrane consists of several components wrapped on the $\mathrm{CP}^{1}$ cycles $C_{i}$. As explained in [3], the number of $\mathrm{CP}^{1}$ components wrapped on $C_{1}, \ldots, C_{N_{f}-1}$ are

$$
\underbrace{N_{c}, \ldots, N_{c}}_{N_{f}-N_{c}}, N_{c}-1, \ldots, 2,1 .
$$

One of the equations describing $C$,

$$
x=\Lambda^{-\left(3 N_{c}-N_{f}\right)} v^{N_{f}},
$$

actually describes the whole configuration projected to the Taub-NUT space. Let us look at this equation in the $i$-th patch $U_{i}$, the neighborhood of the $i$-th D6-brane. The equation reads $y_{i}^{N_{f}-i} x_{i}^{N_{f}+1-i}=\Lambda^{-\left(3 N_{c}-N_{f}\right)} y_{i}^{N_{c}} x_{i}^{N_{c}}$. If $i$ is smaller than or equal to $N_{f}-N_{c}$, the right hand side has lower powers of $y_{i}$ and $x_{i}$, and the equation splits to $y_{i}^{N_{c}}=0, x_{i}^{N_{c}}=0$ and $y_{i}^{\left(N_{f}-N_{c}\right)-i} x_{i}^{\left(N_{f}-N_{c}+1\right)-i}=\Lambda^{-\left(3 N_{c}-N_{f}\right)}$. From the first and the second equations, we see that the fivebrane wraps $N_{c}$ times on $C_{i}\left(i \leq N_{f}-N_{c}\right)$. The solution of the last equation is the infinite component $C$. If $i$ is larger than $N_{f}-N_{c}$, the left hand side has lower powers, and the equation splits to $y_{i}^{N_{f}-i}=0, x_{i}^{N_{f}+1-i}=0$, and $y_{i}^{i-\left(N_{f}-N_{c}\right)} x_{i}^{i-\left(N_{f}-N_{c}+1\right)}=\Lambda^{3 N_{c}-N_{f}}$. We see that the fivebrane wraps $\left(N_{f}-i\right)$-times on $C_{i}\left(i>N_{f}-N_{c}\right)$ from the first and the second equation. The solution of the last equation is again the infinite component $C$. The solution to $y_{1}^{N_{c}}=0$ in the first patch is the projection of the other infinite component $C^{\prime}$.

We depict in Figure 2 the fivebrane configuration at the origin of the moduli space (for the case with $N_{f}>N_{c}$ ). The $\mathrm{CP}^{1}$ components can move in the $x^{7,8,9}$ directions and the self-dual two form on them can be turned on. The component $C^{\prime}$ can also be deformed by turning on $M_{i}$ in (2.15). These deformations of the curve correspond to the flat directions of the field theory. ${ }^{1}$

\footnotetext{
${ }^{1}$ It is a matter of question whether the component $C^{\prime}$ can be translated in the $x^{7,8,9}$ directions and
} 


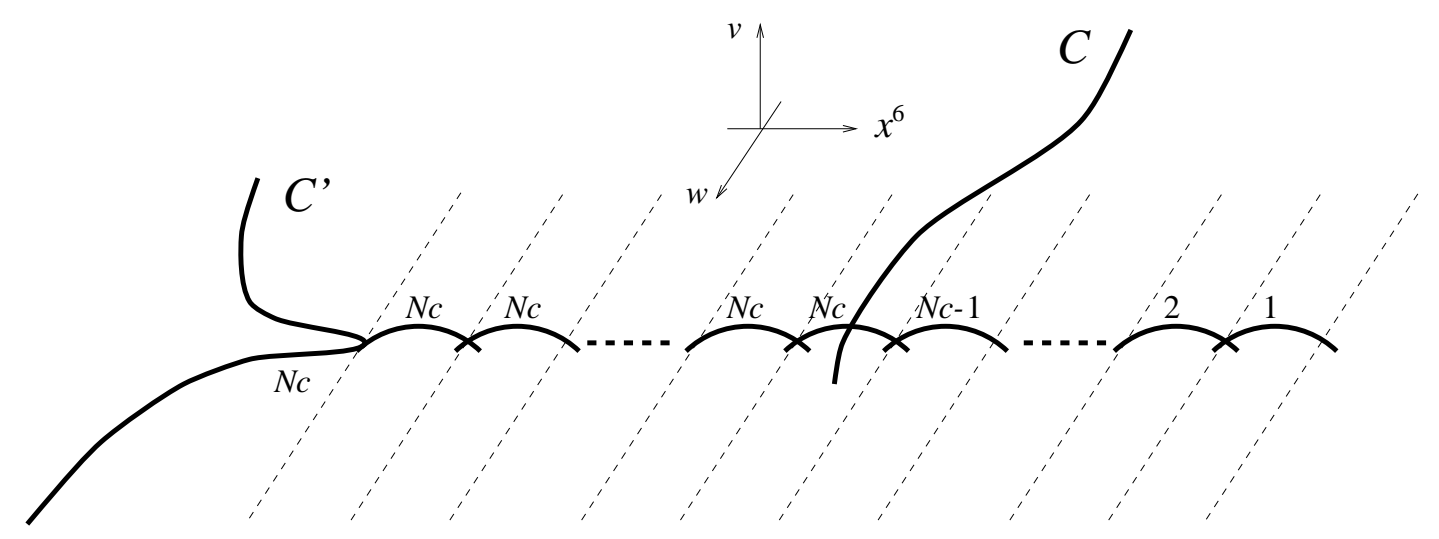

Figure 2: The $M$ Theory Configuration of SQCD

\subsection{The Electric Limit}

Let us consider the limit $\Lambda^{3 N_{c}-N_{f}} \ll E^{3 N_{c}-N_{f}}$ for a fixed energy $E$ and look at the fivebrane configuration with respect to the scales set by $v \sim E$ and $w \sim E^{2}$. (Here we put $Z=1$ by redefining $M_{i}$ and $\Lambda$ so that the quark superfields have canonically normalized kinetic term at the energy $E$.) We also take the limit $R \ll L$ for a fixed length scale $L$ keeping $\mathrm{e}^{L / R}(\Lambda / E)^{3 N_{c}-N_{f}}$ to be of order 1 . In the weakly coupled Type IIA limit $R \ll \ell_{11}$ with $E<1 / \ell_{s t}, 1 / L$, we will see an intersecting Type IIA brane configuration which corresponds to four-dimensional gauge theory. The resulting configuration depends on how to tune the parameter $c$ in the limit. We first consider $c$ to be fixed so that the Taub-NUT space remains well-described by the coordinates $\left(y_{i}, x_{i}\right)$ except that the angular direction $y_{i} \rightarrow \mathrm{e}^{i \theta} y_{i}, x_{i} \rightarrow \mathrm{e}^{-i \theta} x_{i}$ shrinks to zero size in the Type IIA limit.

As we have seen, the infinite component $C$ is described in the $i$-th patch by

$$
\begin{aligned}
& y_{i}^{\left(N_{f}-N_{c}\right)-i} x_{i}^{\left(N_{f}-N_{c}+1\right)-i}=\Lambda^{-\left(3 N_{c}-N_{f}\right)}, \text { for } 1 \leq i \leq N_{f}-N_{c} \\
& y_{i}^{i-\left(N_{f}-N_{c}\right)} x_{i}^{i-\left(N_{f}-N_{c}+1\right)}=\Lambda^{3 N_{c}-N_{f}}, \text { for } N_{f}-N_{c}<i \leq N_{f} .
\end{aligned}
$$

Let us see how this curve behaves in the limit $\Lambda^{3 N_{c}-N_{f}} \rightarrow 0$ in each patch:

$\left(i \leq N_{f}-N_{c}\right)$ : Equation (3.5) shows that either $y_{i}$ or $x_{i}$ diverges. Namely, the curve $C$ goes away from this patch.

whether its self-dual two form can be turned on ( $x^{8,9}$ translation corresponds to turning on $\left.\sum_{i} M_{i}\right)$, since it appears to cost an infinite amount of energy due to the non-compactness of the component $C^{\prime}$. The moduli space of the worldvolume theory have the same dimension as the field theory moduli space only when these degrees of freedom are considered as moduli but not as parameters. 
$\left(i=N_{f}-N_{c}+1\right)$ : Equation (3.6) reads $y_{i}=\Lambda^{3 N_{c}-N_{f}}$, which becomes $y_{i}=0$ in the limit. Namely, the curve $C$ wraps once on the $\mathrm{CP}^{1}$ cycle $C_{i}=C_{N_{f}-N_{c}+1}$.

$\left(i=N_{f}-N_{c}+2\right)$ : Equation (3.6) reads $y_{i}^{2} x_{i}=\Lambda^{3 N_{c}-N_{f}}$. As $\Lambda^{3 N_{c}-N_{f}}$ is sent small, for a fixed non-zero $y_{i}$, the $x_{i}$ value becomes small. On the other hand, for a fixed non-zero $x_{i}$, the two branches of $y_{i}$ value both become small. In the limit $\Lambda^{3 N_{c}-N_{f}} \rightarrow 0$, the curve $C$ wraps once on $C_{i-1}=C_{N_{f}-N_{c}+1}$ as we have seen, and twice on $C_{i}=C_{N_{f}-N_{c}+2}$.

$\left(i>N_{f}-N_{c}+1\right.$ in general): Equation (3.6) shows that, in the limit, the curve $C$ wraps $i-\left(N_{f}-N_{c}+1\right)$ times on $C_{i-1}$ and $i-\left(N_{f}-N_{c}\right)$ times on $C_{i}$.

Thus, in the strict $\Lambda^{3 N_{c}-N_{f}}=0$ limit, the component $C$ wraps on the $\mathbf{C P}^{1}$ cycles $C_{1}, \ldots, C_{N_{f}-1}$ with the following multiplicity ${ }^{1}$

$$
\underbrace{0, \ldots, 0}_{N_{f}-N_{c}}, 1,2, \ldots, N_{c}-1
$$

If we look at the last patch $\left(i=N_{f}\right)$, we see that the curve wraps $N_{c}$-times the locus of $y_{N_{f}}=0$. This locus is the $x$ axis which looks like a semi-infinite cigar. In particular, it appears that the curve becomes infinitely elongated and the worldvolume theory can no longer be considered as a four-dimensional theory. However, we recall here that we are taking the limit $R \rightarrow 0$ at the same time by keeping $\Lambda^{3 N_{c}-N_{f}} \mathrm{e}^{L / R}=E^{3 N_{c}-N_{f}}$ finite for some $L$. Then, the $v$ value starts growing exponentially at some value of $x^{6}$ around $x^{6}=L$ in the scale set by $E$. Beyond such values of $x^{6}$, gluons and gluinos have a large mass compared to $E$, and does not contribute to the dynamics.

Together with the other infinite component $C^{\prime}$ and the original $\mathrm{CP}^{1}$ components, we find that the configuration of the fivebrane looks as in Figure 3. In the Type IIA limit, this is nothing but the configuration of [7] describing the $N=1$ SQCD with gauge group $S U\left(N_{c}\right)$ and $N_{f}$ flavors.

Actually, we have not carefully looked at the behaviour of the component $C^{\prime}$ in the limit. The component $C^{\prime}$ starts blowing up in the $w$ direction at $x^{6}=-L^{\prime}$ where $L^{\prime}$ is given by $c \mathrm{e}^{L^{\prime} / R}{\sqrt{2 L^{\prime}}}^{N_{f}} \sim E^{2 N_{c}}$ (for simplicity we have put the D6-branes on top of each other). If we fix the constant $c$ as in the discussion above, $L^{\prime}$ approaches zero as $R \rightarrow 0$ and therefore the component $C^{\prime}$ becomes close to the D6-branes. ${ }^{2}$ If we want to have a configuration as depicted in Figure 3, we must take a limit $c \rightarrow 0$ such that

\footnotetext{
${ }^{1}$ This argument was used in [3] to obtain this configuration for $N=1$ SQCD by rotating the $N=2$ configuration by an angle $\mu$ and taking the $\mu \rightarrow \infty$ limit.

${ }^{2}$ It is interesting to note that this limit leads to a configuration where an $\mathrm{NS}^{\prime}$ brane divides the D6branes into two parts. This is the configuration to which the chiral flavor symmetry is attributed in $[25]$.
} 


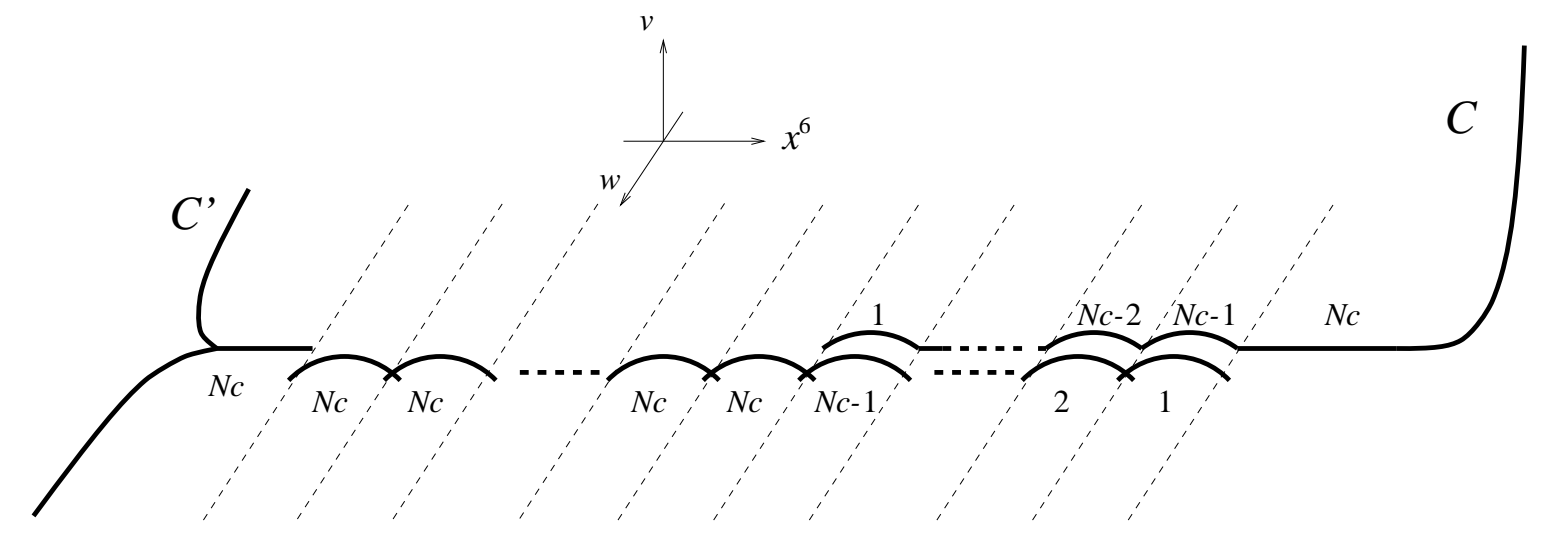

Figure 3: The Electric Limit. (The original $\mathrm{CP}^{1}$ components have been moved from $\vec{w}=0$ in order to avoid confusion with the new ones.)

$c \mathrm{e}^{L^{\prime} / R}{\sqrt{2 L^{\prime}}}^{N_{f}} \sim E^{2 N_{c}}$ holds for fixed $L^{\prime}$ and $E$. In such a limit, $y$ and $x$ degenerates even away from the sixbranes and the Taub-NUT space is no longer well-described by the coordinates $\left(y_{i}, x_{i}\right)$ defined by (3.1)-(3.2). However, we can rescale the coordinates so that the configuration is still described by (3.5) and (3.6) with $\Lambda^{3 N_{c}-N_{f}}$ being replaced by $c^{-1} \Lambda^{3 N_{c}-N_{f}}$. Therefore, as far as $c^{-1} \Lambda^{3 N_{c}-N_{f}}$ becomes zero in the limit (i.e. as long as $L^{\prime}$ does not exceed $L$ ), the behaviour of the other component $C$ remains the same and we obtain the configuration as depicted in Figure 3. Things are different if $c \rightarrow 0$ is taken so that $c^{-1} \Lambda^{3 N_{c}-N_{f}}$ does not become zero; for example, if it blows up. It will become clear what we will obtain in such a limit once we consider the other limit, $\Lambda^{3 N_{c}-N_{f}} \rightarrow \infty$ as we will do shortly.

The above configuration was obtained in [3] by rotating the configuration for asymptotic free $N=2 \mathrm{SQCD}$ and therefore, strictly speaking, one can claim only for $N_{f}<2 N_{c}$ that it corresponds to SQCD. However, it is clear from what has been observed (identification of the gauge coupling constant and the existence of correct Type IIA limit), that it applies also for $2 N_{c} \leq N_{f}<3 N_{c}$. This is actually true even for larger number of flavors $N_{f}>3 N_{c}$. In either case, if $\Lambda^{3 N_{c}-N_{f}} \ll E^{3 N_{c}-N_{f}}$ the gauge theory on the brane is weakly coupled at the energy $E<1 / \ell_{s t} \ll 1 / R$ where extra degrees of freedom are heavy, and therefore the configuration does indeed correspond to SQCD. For $N_{f}>3 N_{c}$, the limit $\Lambda^{3 N_{c}-N_{f}} \ll E^{3 N_{c}-N_{f}}$ corresponds to $E \ll \Lambda$. In fact, $\Lambda$ in such a case is considered as the Landau pole of the infra-red free theory, and hence at energies much smaller than $\Lambda$ we have weakly coupled gauge theory. 


\subsection{The Magnetic Limit}

Let us consider the opposite limit $\Lambda^{3 N_{c}-N_{f}} \rightarrow \infty$ and look at the configuration with respect to some fixed scales in the $v$ and $w$ directions.

From (3.5) and (3.6), we see that the component $C$ behaves in the limit $\Lambda^{3 N_{c}-N_{f}} \rightarrow \infty$ as follows:

$\left(i<N_{f}-N_{c}\right)$ : Equation (3.5) shows that, in the limit, the curve $C$ wraps $N_{f}-N_{c}+1-i$ times on $C_{i-1}$ and $N_{f}-N_{c}-i$ times on $C_{i}$.

$\left(i=N_{f}-N_{c}\right)$ : Equation (3.5), $x_{i}=\Lambda^{-\left(3 N_{c}-N_{f}\right)}$, becomes $x_{i}=0$ in the limit, showing that the curve $C$ wraps once on $C_{i-1}=C_{N_{f}-N_{c}-1}$.

$\left(i>N_{f}-N_{c}\right)$ : Equation (3.6) shows that either $y_{i}$ or $x_{i}$ diverges. This means that the component $C$ goes away from this patch.

Thus, in the strict limit $\Lambda^{3 N_{c}-N_{f}}=\infty$, the component $C$ wraps on the $\mathrm{CP}^{1}$ cycles with the multiplicity

$$
N_{f}-N_{c}-1, \ldots, 2,1, \underbrace{0, \ldots, 0}_{N_{c}}
$$

If we look at the first patch $(i=1)$, it appears that the component $C$ wraps $N_{f}-N_{c}$ times on the $y$ axis (described by $x_{1}=0$ ) and becomes infinitely elongated in the limit $\Lambda^{3 N_{c}-N_{f}} \rightarrow \infty$. However, if we take also the limit $R \rightarrow 0$ at the same time by keeping fixed $\Lambda^{-\left(3 N_{c}-N_{f}\right)} \mathrm{e}^{L / R}$ for some $L$, the value of $v$ starts growing exponentially at some value of $x^{6}$ around $x^{6}=-L$.

Together with the component $C^{\prime}$ and the original $\mathbf{C P}^{1}$ components, we see that the fivebrane configuration in the limit looks as in Figure 4. Here again, in order for the component $C^{\prime}$ to look as in Figure 4, we need to tune the parameter $c$ as $c \rightarrow 0$ as indicated in the electric limit. If we keep $c$ fixed, the component $C^{\prime}$ approaches the sixbranes.

In the weakly coupled Type IIA limit, the configuration in Figure 4 is nothing but the one of [7] describing the magnetic theory. In particular, we can recognize the $S U\left(\widetilde{N}_{c}\right)$ gauge symmetry in a part of the component $C$, namely, in the region sandwiched between the part of $C$ with large values of $v$ (which is identified with the NS 5-brane) and the part of $C^{\prime}$ with large values of $w$ (which is identified with the $\mathrm{NS}^{\prime} 5$-brane). The rest of the curve, to the right of the $\mathrm{NS}^{\prime}$ part of $C^{\prime}$, are identified with the D4-branes representing the singlet meson field.

Unlike in the brane motion of Type IIA configuration [7], we do not have to turn on and 


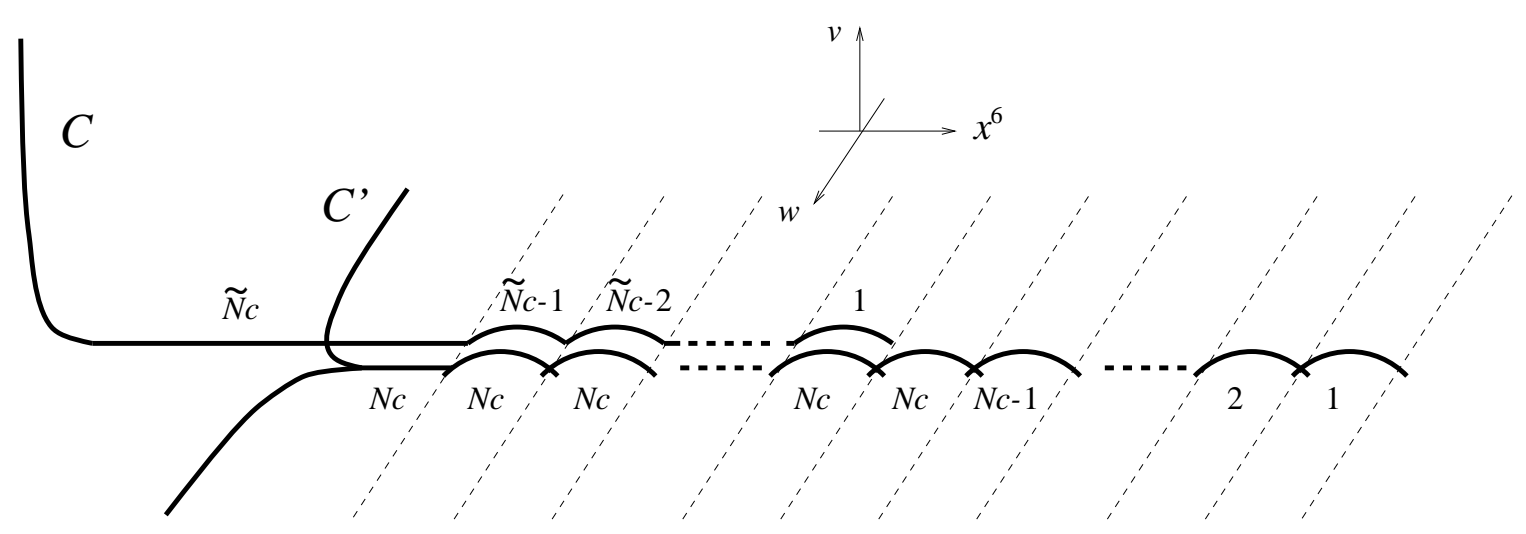

Figure 4: The Magnetic Limit. (The component $C^{\prime}$ has also been moved from $\vec{w}=0$ to avoid confusion with the component $C$.)

off the "Fayet-Iliopoulos parameter" to avoid the intersection of the NS and NS' fivebranes. The components $C$ and $C^{\prime}$ auotomatically avoid each other and are well-separated in the process before taking the Type IIA limit.

\section{The Magnetic Coupling}

In order to measure the $S U\left(\widetilde{N}_{c}\right)$ gauge coupling constant, we must be more precise about the identification of the regions of $C$ and $C^{\prime}$ with the NS, D4 and $\mathrm{NS}^{\prime}$ branes. For this, we need to set an energy scale $E$.

We start with identifying the $N_{f}$ D4-branes stretched between $\mathrm{NS}^{\prime}$ and the left most D6-brane. We see from Figure 4 that $N_{c}$ of them come from $C^{\prime}$ and $\widetilde{N}_{c}$ from $C$. Motion of these D4-branes in the $w$-direction corresponds to the fluctuation of the $N_{f}$ eigenvalues of the gauge singlet meson. Those from $C$ are stuck at $w=0$ but those from $C^{\prime}$ varies as a function of $y$ as $w \sim y^{1 / N_{c}}$. The singlet meson is light compared to the mass scale $E$ when the separation of the eigenvalues are smaller than $E$. Therefore, we may identify the $\widetilde{N}_{c}$ of the D4-branes under question as the part in $C^{\prime}$ with $|w| \leq \mu E$, where we have introduced a constant mass scale $\mu$ to match the dimension. In other words, it is the part with $|y|<\left|y^{\prime}(E)\right|$ where $y^{\prime}(E)=(\mu E)^{N_{c}}$. For this region of $y$, the distance between the two components $C$ and $C^{\prime}$ in the $v$ direction is much smaller than their distance in the $w$ direction in the weakly coupled Type IIA limit. Therefore, we can identify the $N_{f}$ D4-branes as the region of the components $C$ and $C^{\prime}$ with $|y|<\left|y^{\prime}(E)\right|$ (except the part of $C$ wrapping the $\mathrm{CP}^{1}$ cycles).

We next distinguish the $\widetilde{N}_{c}$ D4-branes from the NS 5-brane in the component $C$. Since 
the scale in the $w$ direction is already set by $w \sim \mu E$, the scale in the $v$-direction is set by $v \sim \mu^{-1} E^{2}$. Thus, the boundary between the NS5-brane and the D4-branes should be at $y=y(E):=\Lambda^{3 N_{c}-N_{f}}\left(\mu^{-1} E^{2}\right)^{N_{f}-N_{c}}$.

Therefore, we can identify the $\widetilde{N}_{c}$ D4-branes responsible for the $S U\left(\widetilde{N}_{c}\right)$ gauge dynamics as the region of the component $C$ with $\left|y^{\prime}(E)\right|<|y|<|y(E)|$. In particular, the gauge coupling constant $\widetilde{g}(E)$ of the magnetic $S U\left(\widetilde{N}_{c}\right)$ theory at the energy $E$ is given by

$$
\mathrm{e}^{-1 / \widetilde{g}^{2}(E)}=\frac{y^{\prime}(E)}{y(E)}=\frac{\mu^{N_{f}} \Lambda^{-\left(3 N_{c}-N_{f}\right)}}{E^{3 \widetilde{N}_{c}-N_{f}}} .
$$

The relation (3.9) implies that the dynamical scale $\widetilde{\Lambda}$ of the magnetic theory is given by

$$
\Lambda^{3 N_{c}-N_{f}} \widetilde{\Lambda}^{3 \widetilde{N}_{c}-N_{f}}=\mu^{N_{f}}
$$

which is a familiar formula in field theory [26].

\section{Holomorphic Description of the Magnetic Theory}

Under a suitable change of the coordinates, we can express the curve purely in terms of the observables of the magnetic theory. From the discussion of the energy scales, it is natural to change the coordinates $w$ and $v$ as

$$
w=\mu \widetilde{w}, \quad v=\mu^{-1} \widetilde{v} .
$$

Accordingly, we also change the coordinates $y$ and $x$ as $y=\mu^{N_{c}} \widetilde{y}, x=\mu^{-\left(N_{c}+N_{f}\right)} \widetilde{x}$. Then, the complex structure of the space-time is described by $\widetilde{y} \widetilde{x}=\widetilde{v}^{N_{f}}$, and the curve is given by

$$
C\left\{\begin{array} { l } 
{ \widetilde { x } = \widetilde { \Lambda } ^ { 3 \widetilde { N } _ { c } - N _ { f } } \widetilde { v } ^ { N _ { c } } } \\
{ \widetilde { w } = 0 , }
\end{array} \quad C ^ { \prime } \left\{\begin{array}{l}
\widetilde{y}=\prod_{i=1}^{N_{c}}\left(\widetilde{w}-\widetilde{M}_{i}\right) \\
\widetilde{v}=0 .
\end{array}\right.\right.
$$

In the above expression, $\widetilde{M}_{i}$ are the eigenvalues of the singlet meson in the magnetic theory which are related to the electric variables $M_{i}$ by

$$
M_{i}=\mu \widetilde{M}_{i}
$$

The fact that there are only $N_{c}$ parameters is consistent with the fact that at most only $N_{c}$ eigenvalues of the singlet meson can have non-zero vevs [8]. By the transformation properties under the rotation in the 45 and 89 planes, ${ }^{1}$ it is natural to identify $\widetilde{v}$ as the

\footnotetext{
${ }^{1}$ We record the $U(1)_{45}$ and $U(1)_{89}$ charges of the parameters and fields:

$$
\begin{array}{cccccccccccc} 
& x(\widetilde{x}) & y(\widetilde{y}) & v(\widetilde{v}) & w(\widetilde{w}) & \Lambda^{3 N_{c}-N_{f}} & \widetilde{Q} Q & B \widetilde{B} & \widetilde{\Lambda}^{3} \widetilde{N}_{c}-N_{f} & q \widetilde{q} & b \widetilde{b} & \mu \\
U(1)_{45} & 2 N_{f} & 0 & 2 & 0 & 2 N_{c}-2 N_{f} & 0 & 0 & 2 \widetilde{N}_{c} & 2 & 2 \widetilde{N}_{c} & 0 \\
U(1)_{89} & -2 N_{c} & 2 N_{c} & 0 & 2 & 2 N_{c} & 2 & 2 N_{c} & -2 N_{c} & 0 & 0 & 0
\end{array}
$$
}


magnetic meson field $q \widetilde{q}$. This leads to an interpretation of our claim that the region of D4-branes in $C$ is in $|v|<\mu^{-1} E^{2}$, namely in $|\widetilde{v}|<E^{2}$; For $|\widetilde{v}|>E^{2}$, the gauge group is broken at an energy above $E$ by the Higgs mechanism and does not contribute to the dynamics. Also, the fact that there is no characteristic parameter in the $v$-direction is consistent with the fact that the vacuum expectation value of the magnetic meson is zero. As in the electric theory, by looking at the $U(1)$ charge, we may identify the magnetic baryon vev with the separation of the values of $\widetilde{\Lambda}^{3 \widetilde{N}_{c}-N_{f}} \widetilde{y}$ at $\widetilde{v}=\widetilde{w}=0$ between the two components $C$ and $C^{\prime}$ :

$$
b \widetilde{b}=\left.\widetilde{\Lambda}^{3 \widetilde{N}_{c}-N_{f}} \Delta \widetilde{y}\right|_{\widetilde{v}=\widetilde{w}=0} .
$$

In the above expression, $b \tilde{b}$ is the combination $\left(1 / \widetilde{N}_{c} !\right) b_{i_{1}, \ldots,{ }_{N_{c}}} \widetilde{b}^{i_{1}, \ldots, \tau_{N_{c}}}$ of the magenetic baryon fields which is invariant under the action of the diagonal subgroup $U\left(N_{f}\right)$ of the chiral flavor symmetry group. By the equations defining $C$ and $C^{\prime}$, we have

$$
b \widetilde{b}=(-1)^{N_{c}} \widetilde{\Lambda}^{3 \widetilde{N}_{c}-N_{f}} \prod_{i=1}^{N_{c}} \widetilde{M}_{i}
$$

which agrees with the quantum modified constraint of the magnetic theory. Actually, this can be considered as a consequence of the relation $B \widetilde{B}:=\left.\Delta y\right|_{v=w=0}=(-1)^{N_{c}} \prod_{i=1}^{N_{c}} M_{i}$ corresponding to the classical constraint of the electric theory under the map [26] of the observables

$$
b \widetilde{b}=\mu^{-N_{c}} \widetilde{\Lambda}^{3 \widetilde{N}_{c}-N_{f}} B \widetilde{B}
$$

which follows from the coordinate transformation $\widetilde{y}=\mu^{-N_{c}} y$.

\section{The Scale $\mu$}

We now determine the value of the scale $\mu$. Let us go back to the problem of distinguishing the D4-branes from the $\mathrm{NS}^{\prime}$ brane in the component $C^{\prime}$. These $N_{c}$ D4-branes, together with the $\widetilde{N}_{c}$ D4-branes from the component $C$, are interpreted as representing the $N_{f}$ eigenvalues of the gauge singlet meson field, which is a chiral multiplet in the adjoint representation of the diagonal flavor group $U\left(N_{f}\right)$. Therefore, it is the same kind of problem as the one of section 2.1 distinguishing the D4-branes from the NS5-branes in the configuration of the $N=2$ super-Yang-Mills theory. As in that case, the D4-branes are identified as the region where the configuration looks like nearly coincident D4-branes compared to the length scale $\ell_{s t}{ }^{2} E$. Since the length in the $w$-direction is measured by $\left|\ell_{11}{ }^{3} \Delta w\right|$, the $N_{c}$ D4-branes corresponds to the region in $C^{\prime}$ with $\left|\ell_{11}{ }^{3} w\right|<\ell_{s t}{ }^{2} E$, namely 
$|w|<\frac{1}{R} E$. This shows that

$$
\mu=\frac{1}{R}
$$

The space-time metric in the 45-89 directions, which is expressed at large $v \gg R / \ell_{s t}^{2}$ as $\left|\ell_{s t}{ }^{2} \mathrm{~d} v\right|^{2}+\left|\ell_{11}{ }^{3} \mathrm{~d} w\right|^{2}$, is expressed as $\left|\ell_{11}^{3} \mathrm{~d} \widetilde{v}\right|^{2}+\left|\ell_{s t}{ }^{2} \mathrm{~d} \widetilde{w}\right|^{2}$ in terms of the magnetic coordinates $\widetilde{v}$ and $\widetilde{w}$ defined by (3.11). Note the symmetry between the two expressions of the metric.

So far, we have been assuming $Z=1$ and also that the magnetic quarks have canonically normalized kinetic term. We can easily relax this conditon as follows. We consider $Z$ to be general, and also the magnetic quarks to have kinetic term $\widetilde{Z}\left(q^{\dagger} q+\widetilde{q} \widetilde{q}^{\dagger}\right)$ at the energy $E$. Then, the scales in the $\widetilde{v}$ and $\widetilde{w}$ directions at this energy are set by $\widetilde{v} \sim \widetilde{Z}^{-1} E^{2}$ and $\widetilde{w} \sim \widetilde{Z} E$. Since the length scale in the $w$-direction is set by $\ell_{s t}{ }^{2} E$, we have $\ell_{s t}{ }^{2} E=\ell_{11}{ }^{3} Z w=\ell_{11}{ }^{3} Z \mu \widetilde{w}$ and therefore the scale $\mu$ is given by $\mu=$ $Z^{-1} \widetilde{Z}^{-1} \frac{1}{R}$. Then the space-time metric in the 45-89 direction at large $v$ is expressed as $\left|\ell_{s t}{ }^{2} Z^{-1} \mathrm{~d} v\right|^{2}+\left|\ell_{11}{ }^{3} Z \mathrm{~d} w\right|^{2}=\left|\ell_{11}{ }^{3} \widetilde{Z} \mathrm{~d} \widetilde{v}\right|^{2}+\left|\ell_{s t}{ }^{2} \widetilde{Z}^{-1} \mathrm{~d} \widetilde{w}\right|^{2}$.

\subsection{Deformations}

\subsubsection{Mass Perturbation of the Electric Theory}

Let us consider turning on a mass term of one of the quarks of the electric theory

$$
W_{\text {tree }}=m \widetilde{Q}^{i_{*}} Q_{i_{*}} \quad\left(\text { no sum over } i_{*}\right)
$$

This corresponds in the brane picture to lifting one of the sixbranes from $v=0$ to $v=-m$. The $\mathrm{W}$-boson stretched between the sixbrane and the D4-branes has a mass $\ell_{s t}{ }^{-2} \Delta\left|x^{4}+i x^{5}\right|=Z^{-1}|m|$. This is nothing but the quark mass under the identification of $Z$ in (2.17) as the normalization factor of the quark kinetic term $\int \mathrm{d}^{4} \theta Z\left(Q^{\dagger} Q+\widetilde{Q} \widetilde{Q}^{\dagger}\right)$.

Suppose we are lifting the $i_{*}$-th sixbrane (from the left) with $1<i_{*}<N_{f}$. Then, the two-cycles $C_{i_{*}-1}$ and $C_{i_{*}}$ defined as the fibres over the segments $\left[\vec{x}_{i_{*}-1}, \vec{x}_{i_{*}}\right]$ and $\left[\vec{x}_{i_{*}}, \vec{x}_{i_{*}+1}\right]$ become tilted and are no longer supersymmetric with respect to the supersymmetry preserved by the fivebrane components wrapped on other cycles. However, the cycle $\widetilde{C}$ defined as the fibres over the straight segment $\left[\vec{x}_{i_{*}-1}, \vec{x}_{i_{*}+1}\right]$ is in the line $v=0$ and is still supersymmetric. This cycle is a smooth two-sphere with a neck in the middle which has a circumference $\sim 4 \pi \ell_{s t}{ }^{2}|m|$ if $\ell_{s t}{ }^{2} m \ll R$. What has been said applies also for $i_{*}=1, N_{f}$ with an obvious modification. 

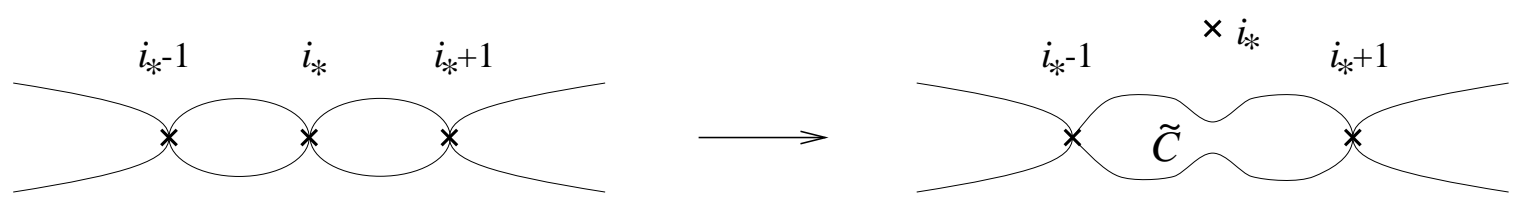

Figure 5: Lifting the $i_{*}$-th Sixbrane

The fivebrane configuration is supersymmetric after the deformation only when the components wrapped on $C_{i_{*}-1}$ and $C_{i_{*}}$ merge into components wrapped on $\widetilde{C}$. This is possible only if the numbers of components wrapped on $C_{i_{*}-1}$ and $C_{i_{*}}$ are equal and their location in the $x^{7,8,9}$ directions match. Therefore we can lift the sixbrane only for $i_{*}=1, \ldots, N_{f}-N_{c}$. In particular we cannot lift any one of the sixbranes when $N_{f}=N_{c}$. This is actually consistent with field theory since giving a mass to a quark would lead to SQCD with $N_{c}-1$ massless quarks which has no supersymmetric stable vacuum [27].

The holomorphic description of the curve after lifting the sixbrane remains the same as (2.15) where $x$ and $y$ now obey the relation

$$
y x=v^{N_{f}-1}(v+m) .
$$

The limit $m \rightarrow \infty$ exists if $\Lambda$ is sent to zero so that $\Lambda_{L}^{3 N_{c}-\left(N_{f}-1\right)}=m \Lambda^{3 N_{c}-N_{f}}$ is kept fixed and if $\hat{x}=m^{-1} x$ becomes a good coordinate. In this case, the expression of the curve (2.15) goes over to the one corresponding to the theory with $N_{f}-1$ flavors whose dynamical scale is $\Lambda_{L}$.

In terms of the real coordinates, $y$ and $x$ are expressed for large $m$ as

$$
\begin{aligned}
& y=\hat{c} \times \mathrm{e}^{-\left(x^{6} / R+i x^{10}\right)} \prod_{i \neq i_{*}} \sqrt{\left|\vec{x}-\vec{x}_{i}\right|-\left(x^{6}-x_{i}^{6}\right)} . \\
& x=m \hat{c}^{-1} \times\left(\frac{v}{\left|x^{4}+i x^{5}\right|}\right)^{N_{f}-1} \mathrm{e}^{x^{6} / R+i x^{10}} \prod_{i \neq i_{*}} \sqrt{\left|\vec{x}-\vec{x}_{i}\right|+\left(x^{6}-x_{i}^{6}\right)},
\end{aligned}
$$

where $\hat{c}=c \ell_{s t} \sqrt{|m| / Z}$. Therefore $\hat{x}$ (and also $y$ ) remains a good coordinates in the limit $m \rightarrow \infty$ if $c$ is sent to zero so that $\hat{c}$ is kept fixed.

It is easy to see the consequence of lifting the sixbrane in the Type IIA limits. In the electric limit, it is just to lift a D6-brane with certain rejoining of the D4-branes (see Figure 6). In the magnetic limit, a D4-brane ending on the lifted sixbrane is created (see Figure $6)$. It is at $\widetilde{v}=-\mu m$ in the magnetic coordinate. This corresponds to the Higgsing of the magnetic theory, $q^{i_{*}} \widetilde{q}_{i_{*}}=-\mu m$, induced by the superpotential $W_{m a g}=\frac{1}{\mu} M q \widetilde{q}+m M_{i_{*}}^{i_{*}}$. 


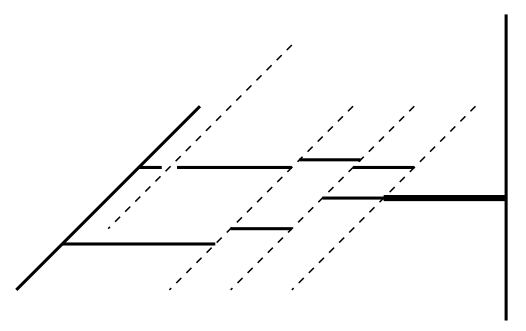

(a)

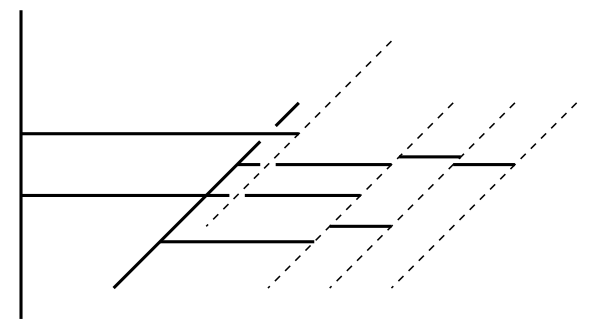

(b)

Figure 6: The Deformation (3.18) in the case $N_{c}=2, N_{f}=4$.

The mass deformation in the electric theory (a) corresponds to the Higgsing in the magnetic theory (b).

This is the brane realization of the correspondence of mass-deformation of the electric theory (making the coupling stronger) and the Higgsing of the magnetic theory (making the coupling weaker).

\subsubsection{Higgsing the Electric Theory}

It is possible to have a supersymmetric configuration if we lift all of the sixbranes from $v=0$ :

$$
y x=\prod_{i=1}^{N_{f}}\left(v+m_{i}\right), \quad m_{i} \neq 0 .
$$

This corresponds in field theory to giving bare mass to all of the quarks, and the low energy physics is that of $S U\left(N_{c}\right)$ super-Yang-Mills theory with the dynamical scale $\Lambda_{L}$ given by $\Lambda_{L}^{3 N_{c}}=m_{1} \cdots m_{N_{f}} \Lambda^{3 N_{c}-N_{f}}$ which exhibits chiral symmetry breaking $\mathbf{Z}_{2 N_{c}} \rightarrow \mathbf{Z}_{2}$. The baryon operators have zero vevs but the meson vev is non-zero, $M=\operatorname{diag}\left(\Lambda_{L}^{3} / m_{i}\right)$. The brane configuration as obtained in $[3,5]$ consists of a single component

$$
\begin{aligned}
v w & =\Lambda_{L}^{3}, \\
x & =\Lambda^{-3 N_{c}+N_{f}} v^{N_{c}} .
\end{aligned}
$$

Indeed, there are $N_{c}$ possible configurations corresponding to the $N_{c}$ solutions for $\Lambda_{L}^{3}$. The result applies also to the case $N_{f}<N_{c}$. The relation between $y$ and $w$ is $y=$ $w^{N_{c}-N_{f}} \prod_{i=1}^{N_{f}}\left(w+\Lambda_{L}^{3} / m_{i}\right)$ and the eigenvalues of the meson matrix appears as the root of $y=0$. In the case $N_{f}<N_{c}$, the meson vev $M=\Lambda_{L}^{3} / m$ follows from the dynamical generation of the superpotential [27] in the field theory side. Thus, one can say that the 
brane configuration encodes an information about the superpotential generation. There is also a similar interpretation for higher flavor cases $N_{f}>N_{c}$.

Let us consider the limit where $N_{c}$ of $m_{i}$ approaches zero as $m_{i}=\alpha \tilde{m}_{i}, \alpha \rightarrow 0$ $\left(i=N_{f}-N_{c}+1, \ldots, N_{f}\right)$ with $\tilde{m}_{i}$ kept finite. Then, $\Lambda_{L}^{3} / m_{i}$ approaches zero for $i=$ $1, \ldots, N_{f}-N_{c}$, but it remains the same for $i=N_{f}-N_{c}+1, \ldots, N_{f}$. In field theory, this leads to a vacuum of SQCD with $N_{c}$ massless and $N_{f}-N_{c}$ massive quarks given by

$$
M=\operatorname{diag}\left(0, \ldots, 0, M_{1}, \ldots, M_{N_{c}}\right), \quad B=\widetilde{B}=0,
$$

where $M_{i}=\Lambda_{L}^{3} / m_{N_{f}-N_{c}+i}$. At this stage, we can send $m_{1}, \ldots, m_{N_{f}-N_{c}}$ to zero keeping fixed $M_{i}$, which yields a vacuum of SQCD with $N_{f}$ massless quarks with the meson and baryon vevs as in (3.25). In the brane picture, the corresponding procedure leads

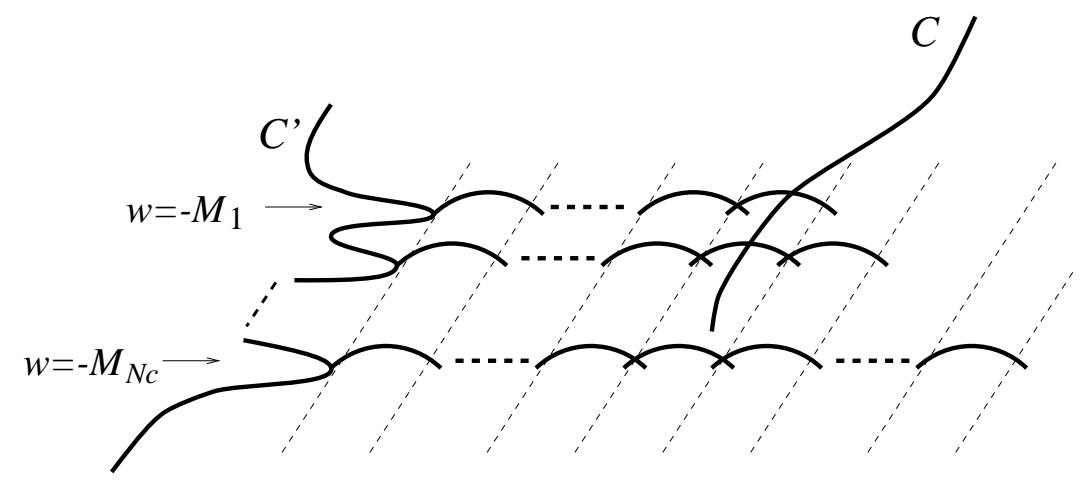

Figure 7: The Configuration Corresponding to the Vacuum (3.25)

to the configuration as depicted in Figure 7 , where the $\mathbf{C P}^{1}$ components make rows at $w=-M_{1}, \ldots,-M_{N_{c}}$.

\section{Decoupling}

Let us consider sending one of the meson eigenvalues, say $M_{N_{c}}$ in (3.25), to infinity:

$$
M_{N_{c}} \longrightarrow \infty
$$

In field theory, this leads to $S U\left(N_{c}-1\right)$ SQCD with $N_{f}-1$ flavors when $\Lambda$ is tuned so that $M_{N_{c}}^{-1} \Lambda^{3 N_{c}-N_{f}}$ is kept fixed. The dynamical scale $\hat{\Lambda}$ of this theory is given by $\hat{\Lambda}^{3\left(N_{c}-1\right)-\left(N_{f}-1\right)}=M_{N_{c}}^{-1} \Lambda^{3 N_{c}-N_{f}}$.

In the brane picture, this corresponds to sending the $N_{c}$-th row at $w=-M_{N_{c}}$ to infinity. One can also send the right-most D6-brane to the infinity $x_{N_{f}}^{6} \rightarrow+\infty$ at the 
same time. Then, it is appropriate to use the coordinates $\hat{y}=M_{N_{c}}^{-1} y$ and $\hat{x}=M_{N_{c}} v^{-1} x$ which are expressed in the limit as (2.12) and (2.13) where $N_{f}$ and $c$ are replaced by $N_{f}-1$ and $\hat{c}=M_{N_{c}}^{-1} \sqrt{2 x_{N_{f}}^{6}} c$. In terms of these coordinates, the expression of the curve (2.15) goes over to the one with $N_{c}, N_{f}, \Lambda$ being replaced by $N_{c}-1, N_{f}-1, \hat{\Lambda}$. The number of components wrapping $C_{i}$ has reduced by one for $i=1, \ldots, N_{f}-2$ while the last $\mathbf{C P}^{1}$ cycle $C_{N_{f}-1}$ has become infinitely elongated and no fivebrane wrapps on it.

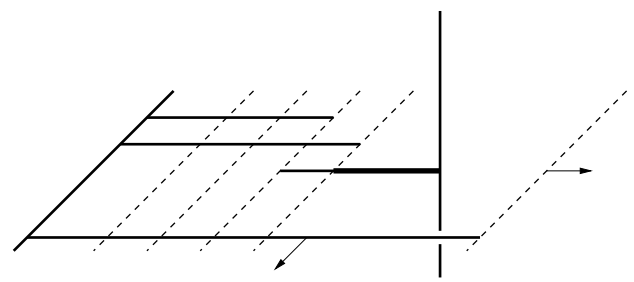

(a)

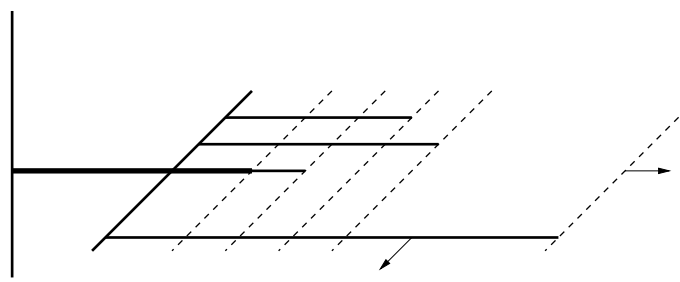

(b)

Figure 8: The Decoupling (3.26).

Higgsing in the electric theory (a) corresponds to giving a mass to a quark in the magnetic theory (b).

In Figure 8, we depict the two Type IIA limits of the above process. In the magnetic limit, the color remains the same but one quark has decoupled, $N_{f} \rightarrow N_{f}-1$, because of the large mass given by the singlet meson vev.

\subsection{Brane Move and Universality Class of the Worldvolume Theory}

In this section, we have seen that the two Type IIA brane configurations describing the electric and magnetic theories of [8] arize from a common configuration of the fivebrane in $M$ theory. The space-time and the configuration depend on, among others, the parameters $R$ and $\Lambda$ or $\widetilde{\Lambda}$ which are related by $\Lambda^{3 N_{c}-N_{f}} \widetilde{\Lambda}^{3 N_{c}-N_{f}} \sim R^{-N_{f}}$. The electric limit arizes by first sending $\Lambda^{3 N_{c}-N_{f}} \ll E^{3 N_{c}-N_{f}}$ in the eleven-dimensional supergravity region $R \gg \ell_{11}$, where $E$ is an energy scale determining the unit of lengths in the $x^{4,5,7,8,9}$ directions, and then scaling down to the weakly coupled Type IIA string theory region $R \ll \ell_{11}$, $E<M_{s t}$ with the ratio $\Lambda / E$ kept fixed. The magnetic limit arizes by first sending $\widetilde{\Lambda}^{3 \widetilde{N}_{c}-N_{f}} \ll E^{3 \widetilde{N}_{c}-N_{f}}$ in the eleven-dimensional supergravity region and then going down to the weakly coupled Type IIA region with the ratio $\widetilde{\Lambda} / E$ kept fixed. The two $M$ theory configurations before the Type IIA limit are smoothly interpolated without a change in the complex structure. 
There have been a lot of works concerning the interpolation of the electric and magnetic configurations by brane moves within the weakly coupled Type IIA region [1]. A move of branes, when it corresponds to a change of parameters of the gauge theory, changes the physics of the worldvolume theory. If the move corresponds to a change of parameters that have no counterpart in four-dimensional gauge theory, then, it is believed that it does not change the physics. An example of such "irrelevant moves" is changing the position of D6-branes in the $x^{6}$-direction relative to each other or with respect to the NS5 and D4-brane system (this fails to be irrelevant in some cases, as we will mention shortly). Another example is changing the relative separation of the two NS5-branes, which corresponds to a change of the gauge coupling constant. When the gauge theory is asymptotic free, or more generally, when the gauge coupling runs at high energy, this just corresponds to a change of the overall scale of the theory and is irrelevant in the infra-red.

Indeed, what is done in the literature [1] is to utilize the latter move to derive the $N=1$ duality. However, in the course of interpolating the electric and magnetic configurations by this move, there is a point at which the NS5-brane intersect with the other NS5-brane or the D6-branes. Thus, this brane move looks as a singular process and it is not obvious whether we can expect that the universality class of the theory remains the same. Actually it was proposed in the very first literature [7] that the singularity in the process may be avoided by another move which corresponds to turning on the Fayet-Iliopoulos parameter of a $U(1)$ factor of the gauge group. It is doubtful whether the gauge group has an $U(1)$ factor or not. However, even if there is a $U(1)$ factor, turning on the FI term moves the theory away from the origin of the moduli space at which most interesting physics is concentrated. In addition, when the gauge group is symplectic or orthogonal groups, which can be realized by introducing an orientifold-plane, there is no room to turn on the FI parameter and the singularity seems unavoidable $[10,11]$.

What we have done in this section is related to this Type IIA brane move since we have changed the parameter $\Lambda$ corresponding to the dynamical scale of the gauge theory. It can be considered as a resolution of the singularity of the Type IIA brane move by going to $M$ theory. The brane move in the supergravity region is completely smooth and we do not have to turn on the FI parameter (which is actually impossible since we are considering the the gauge symmetry to be $S U\left(N_{c}\right)$ ). We will see in the following sections that the method also applies effectively to the case of symplectic or orthogonal gauge groups where FI term never exists: the singularity which is inevitable in the Type IIA move is resolved by going to $M$ theory. Also, we have mainly considered the case where the quark mass terms are turned off. The mass term, as the FI term, would take the theory away from the interesting point. 
Does the universality class of the theory remain the same? The most subtle point is of course the strong string coupling limit $g_{s t} \gg 1$ at the starting point of the interpolation, and the limit $R \ll \ell_{11}$ in the final stage. As $g_{s t}$ is increased, degrees of freedom other than the gauge fields starts strongly coupled and the theory is no more a simple gauge theory. Nevertheless, it is true that a four-dimensional supersymmetric field theory exists in the infra-red limit at an arbitrary values of $g_{s t}$ : No matter how large it is, if one look at the configuration from an extremely long distance, it looks the same as the starting Type IIA configuration as far as we scale up everything as indicated above. The universality class of a theory is a discrete notion unless there is a parameter coupled to a marginal operator. In supersymmetric theories, such a parameter is usually a complex parameter that can enter into the superpotential. In the present case, the parameters we are changing are $R$ and $c$ which are independent real parameters. ${ }^{1}$ Therefore, these cannot enter into the superpotential but only in the Kähler potential. Then, as long as the process of interpolation is smooth, it is highly possible that there is no singular change in the Kähler potential, and we can expect that the universality class remains the same. The fact that we can identify the gauge coupling constant that fits with NSVZ exact beta function suggests that this worldvolume theory is smoothly connected to the ordinary SQCD or its magnetic dual. Therefore, there is a high possibility that the universality class is constant throughout the process of interpolation.

\section{A General Condition}

One thing we are really interested in is under what kind of brane move the universality class of the worldvolume theory remain unchanged. From what we have argued, we propose the following general condition: Two theories that are realized by Type IIA brane configurations are equivalent in the infra-red limit when they are smoothly interpolated by a family of configurations of $M$ theory fivebrane whose complex structure is constant through the process of interpolation, where the family is parametrized by real parameters.

For illustration, let us consider moves of D6-branes of the electric Type IIA configuration in the $x^{6}$-direction (see Figure 9).

(1) Suppose we change the separation of two neighboring D6-branes, which looks like a smooth move even in Type IIA region. This corresponds to simply changing the size of the $\mathbf{C P}^{1}$-cycle in the $M$ theory configuration, and hence is a smooth deformation of

\footnotetext{
${ }^{1}$ The situation would be different if one other direction of the space-time, say $x^{7}$, is compactified on a circle. Then, its radius can combine with the parameter $R$ to make a complex parameter and the theory can continuously depend on it.
} 


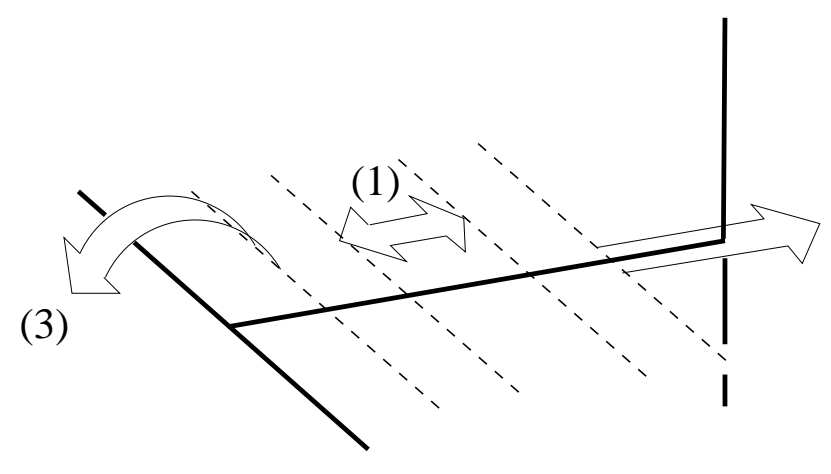

Figure 9: Moving D6-branes

the curve with no change of the complex structure. The size of $\mathrm{CP}^{1}$ is of course a real parameter. (This again fails when we compactify another direction on a circle; the period of the three-form in $M$ theory becomes a complex partner.) Therefore, it does not change the universality class. Indeed, the Kähler metric of the Higgs branch of $N=2$ theories was computed in [6]. The metric explicitly depends on the separation of sixbranes, but the relevant information - the information about the singularity — is independent. (In this particular example, it can explicitly be shown that the dependence on the separation disappears in the weakly coupled Type IIA limit $R / \ell_{11} \rightarrow 0$.)

(2) Let us next consider moving the right-most D6-brane to the right of the NS5-brane. Then, the D6-brane necessarily intersect with the NS5-brane and a single D4-brane stretched between them is created. This looks like a singular process. In $M$ theory configuration, this corresponds to changing the real parameter $c$ in the definition (2.12) of the complex coordinates $y$ and $x$, and hence is a smooth deformation of the curve without any change in the complex structure. The brane "creation" in Type IIA simply corresponds to an approximate wrapping of the fivebrane on the $\mathrm{CP}^{1}$ cycle, and is indeed a smooth process. Nothing is actually created, and there is no singularity nor topology change. Therefore, it does not change the universality class.

(3) Finally, let us move the left-most D6-brane to the left of the NS'5-brane. If we keep the D6-brane at $x^{4,5}=0$ through the process, it must coincide with the $\mathrm{NS}^{\prime} 5$-brane and looks singular. Indeed, an analogous move in $M$ theory is singular as well. However, one could move the D6-brane around the $\mathrm{NS}^{\prime} 5$-brane by turning on and off the mass parameter. Namely, first lift the D6-brane to $x^{4,5} \neq 0$, move it to the left in the $x^{6}$-direction, and then move it down to $x^{4,5}=0$. This looks equally smooth compared to the move used in "deriving" the $N=1$ duality within the Type IIA region: turning on and off the FI 
parameter. It is possible to do the similar move in the $M$ theory configuration. However, it is actually not a smooth move and it also changes the complex structure. The singularity occurs at the very beginning of the move. When the mass is turned on the topology of the configuration changes, as we can see by looking at Figure 5. After that, the configuration is smoothly deformed without any change of the complex structure. Therefore, the total move may change the universality class. Actually, the configuration after the move is smoothly interpolated to the configuration with one less flavors without change of the complex structure, by sending the D6-brane to $x^{6} \rightarrow-\infty$. Thus, the universality class after the move is the same as the one of the theory with $N_{f}-1$ flavors.

\section{Symplectic Groups}

In this section, we generalize the above consideration to SQCD with symplectic gauge groups. We show that the fivebrane configuration has two Type IIA limits corresponding to the electric and magnetic descriptions. Also, we show that the fivebrane configuration proposed in [20] for the dynamical supersymmetry breaking model of [30,31] (IYIT model) has the correct Type IIA limit.

\subsection{SQCD with Symplectic Gauge Group}

In order to realize symplectic gauge group, we consider $M$ theory background that reduces in the Type IIA limit to orientifold four-plane. In particular, we consider $M$ theory on $\mathbf{R}^{4} \times M^{7} / \mathbf{Z}_{2}$ where $M^{7}$ is a product of the Taub-NUT space of $A_{2 N_{f}-1}$ type (described by $y, x, v$ as in the previous sections) and $\mathbf{R}^{3}$ (parametrized by $x^{7}$ and $w \propto$ $\left.x^{8}+i x^{9}\right)$, and the $\mathbf{Z}_{2}$ acts as $y \rightarrow y, x \rightarrow x, v \rightarrow-v$ and $x^{7,8,9} \rightarrow-x^{7,8,9}$. The Taub-NUT space is covered with $2 N_{f}$ coordinate patches as explained in the previous section, and the $\mathbf{Z}_{2}$ acts on the coordinates as $\left(y_{i}, x_{i}\right) \rightarrow\left((-1)^{i-1} y_{i},(-1)^{i} x_{i}\right)$. In particular, it fixes the $\mathrm{CP}^{1}$ cycles $C_{2 i}$ point-wisely, but acts on $C_{2 i+1}$ as the $\pi$-rotation around the intersection points with $C_{2 i}$ and $C_{2 i+2}$.

\section{The Fivebrane Configuration}

The fivebrane configuration for $S p\left(N_{c}\right)$ SQCD with $N_{f}>N_{c}$ flavors can be obatined by rotating the $N=2$ configuration of [28]. ${ }^{1}$

\footnotetext{
${ }^{1}$ This was essentially done in [20], but there was a subtle error in the starting $N=2$ configuration. Here we start with the correct one [28] and present the correct result for $N=1$ SQCD.
} 
The fivebrane consists of two infinite components in $x^{7}=0$

$$
C^{\prime}\left\{\begin{array} { l } 
{ y = \prod _ { i = 1 } ^ { N _ { c } + 1 } ( w ^ { 2 } - M _ { i } ^ { 2 } ) } \\
{ v = 0 , }
\end{array} \quad C \left\{\begin{array}{l}
x=\Lambda^{-\left(6\left(N_{c}+1\right)-2 N_{f}\right)} v^{2\left(N_{c}+1\right)} \\
w=0,
\end{array}\right.\right.
$$

and several $\mathrm{CP}^{1}$ components wrapping the cycles $C_{1}, \ldots, C_{2 N_{f}-1}$. The number of fivebranes wrapping the cycle $C_{i}$ is $2 N_{c}+1$ for odd $i$ and $2 N_{c}+2$ for even $i$ if $1 \leq i \leq$ $2 N_{f}-2 N_{c}-2$, and it is $2\left[\left(2 N_{f}-i\right) / 2\right]$ for larger $i, 2 N_{f}-2 N_{c}-1 \leq i \leq 2 N_{f}-1$. The infinite component $C$ intersects transversely with the $y$-axis at $y=\Lambda^{4\left(N_{c}+1\right)}$ if $N_{f}=N_{c}+1$ while it intersects with the $\mathrm{CP}^{1}$ cycle $C_{2 N_{f}-2 N_{c}-2}$ if $N_{f}>N_{c}+1$. In [28], it was shown that the Dirac quatization condition is not satisfied when the fivebrane in $\mathbf{R}^{5} / \mathbf{Z}_{2}$ orbifold intersects with the $\mathbf{Z}_{2}$ fixed plane transversely in a four-dimensional factor of the space-time. In other words, such an intersection, which we call a t-configuration [20], is forbidden. In order to avoid a $t$-configuration, for $N_{f}=N_{c}+1$, the intersection points of $C$ and $C^{\prime}$ with the $y$-axis in $x^{7,8,9}=0$ must coincide, which requires $\prod_{i=1}^{N_{c}+1}\left(-M_{i}^{2}\right)=\Lambda^{4\left(N_{c}+1\right)}$. For $N_{f}>N_{c}+1$, since the number of fivebranes wrapping $C_{1}$ is odd, the component $C^{\prime}$ must intersects with the $y$-axis in $x^{7,8,9}=0$ at $y=0$, which means that at least one $M_{i}$ must be zero. Also, in the latter case, at least one pair of the $\mathrm{CP}^{1}$ components wrapping $C_{2 i}$ must be at $x^{7,8,9}=0$ for each $1 \leq 2 i \leq 2 N_{f}-2 N_{c}-2$ (see Figure 10).

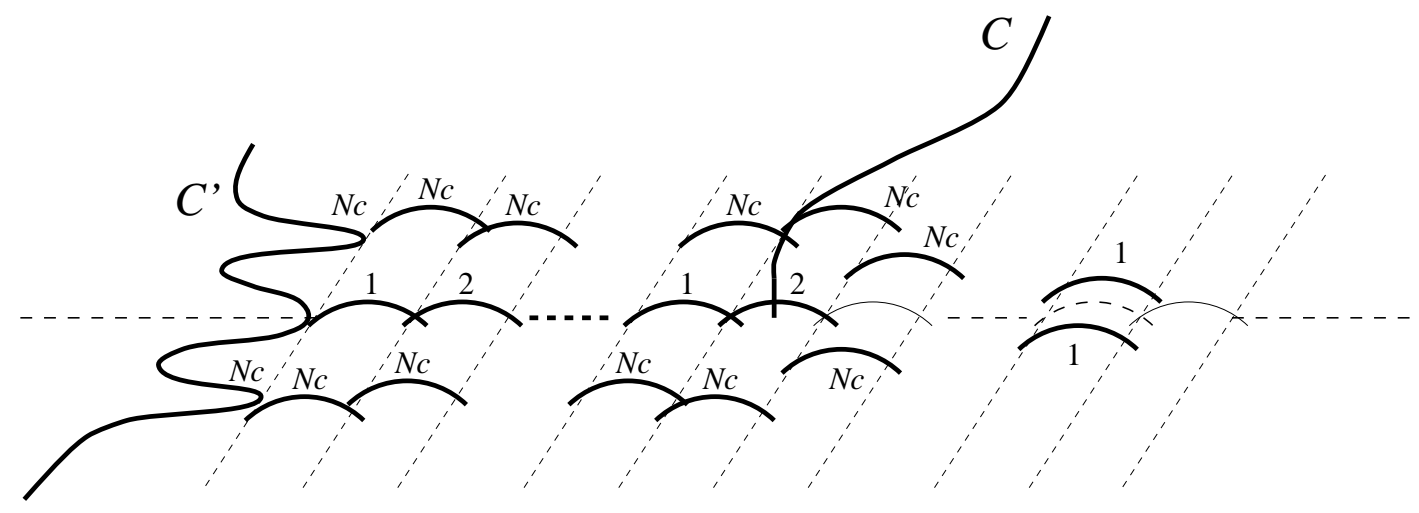

Figure 10: The $M$ Theory Configuration of $S p\left(N_{c}\right)$ SQCD.

Half of the component $C$ is not written here in order to avoid complication. There are $2 N_{f}$ sixbranes. The dashed horizontal lines and curves correspond to the $\mathbf{Z}_{2}$ fixed point set whereas the thin curves stand for $\mathbf{C P}^{1}$ cycles on which the $\mathbf{Z}_{2}$ acts as the $\pi$-rotation.

The parameters $\pm M_{i}$ in (4.1) are interpreted as the eigenvalues of the vev of the meson matrix $M^{i j}=Q_{a}^{i} J^{a b} Q_{b}^{j}$ ( $J^{a b}$ is the $S p\left(N_{c}\right)$ invariant skew-symmetric form) and the above constraint on $M_{i}$ is equivalent to the quantum constraint on $M^{i j}$ in field theory. 
The number of $\mathbf{Z}_{2}$ invariant deformations of the curve subject to the constraints to avoid $t$-configurations agrees with the dimension $4 N_{f} N_{c}-\left(2 N_{c}^{2}+N_{c}\right)$ of the moduli space of vacua of the field theory.

The complex coordinates $y, x, v$ and $w$ are related to the real coordinates $x^{\mu}$ as (2.12), (2.13) and (2.17) where $N_{f}$ is now replaced by $2 N_{f}$. In addition to the parameter and observables of SQCD, the configuration depends on two real parameters $c Z^{2 N_{c}+2} \mathrm{e}^{-\sum_{i} x_{i}^{6} / R}$ and $R$ as well as the separation between the sixbranes.

\section{The Electric Limit}

Let us consider the limit $\Lambda^{3\left(N_{c}+1\right)-N_{f}} \ll E^{3\left(N_{c}+1\right)-N_{f}}$ for a fixed energy scale $E$ and look at the fivebrane with respect to the scale set by $v \sim E, w \sim E^{2}$. The component $C$ is sent to the right and wraps on the $\mathrm{CP}^{1}$ cycles $C_{2 N_{f}-2 N_{c}-1}, \ldots, C_{2 N_{f}-1}$ with multiplicities $1,2, \ldots, 2 N_{c}+1$. It also wraps on the $x$-axis with mulitplicity $2 N_{c}+2$ and then blows up in the $v$-direction at $y(E)=\Lambda^{6\left(N_{c}+1\right)-2 N_{f}} E^{2 N_{f}-2 N_{c}-2}$. The component $C^{\prime}$ wraps on the $y$-axis and blows up in the $w$-direction at $y^{\prime}(E)=E^{4\left(N_{c}+1\right)}$. The two regions with large $v \gg E$ and large $w \gg E^{2}$ are interpreted as the NS5 and NS'5-branes in the Type IIA limit. Between them, the fivebrane wraps $2 N_{c}+2$ or $2 N_{c}+1$ times on the eleventh direction $-2 N_{c}+2$ on cycles point-wisely $\mathbf{Z}_{2}$ invariant and $2 N_{c}+1$ on cycles on which $\mathbf{Z}_{2}$ acts as the $\pi$-rotation. In the weakly coupled Type IIA limit $R \ll \ell_{11}$, a pair of fivebranes wrapping each of the $\mathbf{Z}_{2}$ fixed cycles $\left(C_{2 i}\right.$ and the $y$ - and $x$-axis at $\left.x^{7,8,9}=0\right)$ is identified with the orientifold four-plane of $S p$-type with trivial RR $U(1)$ gauge field (which we denote by $\left.\mathrm{O}^{+}\right)$. Similarly, a single fivebrane wrapped on each of the cycles $C_{2 i+1}$ at $x^{7,8,9}=0$ on which $\mathbf{Z}_{2}$ acts as the $\pi$-rotation is identified with the $S p$-type O4-plane with a non-trivial RR Wilson line [28] (which we denote by $\widetilde{\mathrm{O}}^{+}$). The rest of the fivebrane, wrapping $2 N_{c}$-times on the eleventh direction, are interpreted as $2 N_{c}$ D4-branes. These together yield $S p\left(N_{c}\right)$ gauge symmetry.

The gauge coupling constant $g(E)$ at the energy $E$ can be read as in the $S U\left(N_{c}\right)$ case. It is given by

$$
\mathrm{e}^{-2 / g^{2}(E)}=\frac{y(E)}{y^{\prime}(E)}=\left(\frac{\Lambda}{E}\right)^{6\left(N_{c}+1\right)-2 N_{f}} .
$$

The factor of two can be explained as in [29] as the consequence of a particular embedding of $\operatorname{Sp}\left(N_{c}\right)$ to $S U\left(2 N_{c}\right)$.

As a summary, we depict in Figure 11 (e) the electric Type IIA limit. Note that the dimension of the moduli space can be counted also by applying the "s-rule" found in [28] to this configuration. 


\section{The Magnetic Limit}

Let us next consider the opposite limit $\Lambda^{3\left(N_{c}+1\right)-N_{f}} \rightarrow \infty$. The component $C$ is sent to the left and wraps on the $\mathrm{CP}^{1}$ cycles $C_{1}, \ldots, C_{2 N_{f}-2 N_{c}-3}$ with multiplicities $2 N_{f}-2 N_{c}-$ $3, \ldots, 2,1$. It also wraps $2 N_{f}-2 N_{c}-2$ times on the $y$-axis and starts blowing up in the $v$-direction at some value of $y$. This value of $y$ is much larger than the value where the component $C^{\prime}$ starts blowing up in the $w$-direction with respect to some scale which wll be specified momentarily. Between the two regions with large $v$ and $w$, the fivebrane wraps $2 N_{f}-2 N_{c}-2$ times on the eleventh direction. In the Type IIA limit, two of them are interpreted as providing a charge +2 to the orientifold fixed plane (consituting the $\mathrm{O} 4^{+}$-plane) while the rest, $2 \widetilde{N}_{c}=2 N_{f}-2 N_{c}-4$ of them, are considered as $2 \widetilde{N}_{c}$ degenerate D4-branes. These together yield $S p\left(\widetilde{N}_{c}\right)$ gauge symmetry. We can measure the gauge coupling at an energy $E$ by setting the corresponding length scales in the $v$ and $w$ directions. As in $S U\left(N_{c}\right)$ case, these length scales are set by $w \sim \mu E$ and $v \sim \mu^{-1} E^{2}$ where $\mu$ is a constant mass scale, and the $S p\left(\widetilde{N}_{c}\right)$ gauge coupling $\widetilde{g}(E)$ is measured as

$$
\mathrm{e}^{-2 / \widetilde{g}^{2}(E)}=\frac{\mu^{2 N_{f}} \Lambda^{-6\left(\widetilde{N}_{c}+1\right)+2 N_{f}}}{E^{6\left(\widetilde{N}_{c}+1\right)-2 N_{f}}} .
$$

This yields the standard relation $\Lambda^{6\left(\widetilde{N}_{c}+1\right)-2 N_{f}} \widetilde{\Lambda}^{6\left(\widetilde{N}_{c}+1\right)-2 N_{f}}=\mu^{2 N_{f}}$ between the dynamical scales of the electric and magnetic theories [32] (up to the sign ambiguity).

On the right of the region of $C^{\prime}$ with large $w \gg \mu E$ (which is identified as the NS'5brane), we have components of the fivebrane wrapping several times on the eleventh direction. The fivebrane wraps $2 N_{f}$-times in the region sandwiched between the $\mathrm{NS}^{\prime} 5$ brane and the left-most D6-brane, while it wraps 2[i/2]-times on the $\mathrm{CP}^{1}$ cycle $C_{2 N_{f}-i}$. In the weakly coupled Type IIA limit, these are all interpreted as the D4-branes stretched between the $\mathrm{NS}^{\prime}$ and the left-most D6-brane or between neighboring D6-branes; the number of times the fivebrane wraps is equal to to the number of stretched D4-branes. The $\mathbf{Z}_{2}$ fixed cycle $C_{2 i}$ at $x^{7,8,9}=0$ is identified with the orientifold four-plane of $S O$-type (O4--plane) whereas the $\mathbf{Z}_{2}$ invariant but non-fixed cycle $C_{2 i+1}$ at $x^{7,8,9}=0$ is identified with the $S O$-type O4-plane with a single D4-brane stuck on it ( $4^{0}$-plane) which has a non-trivial RR Wislon line [28]. The stretched D4-branes represent the degrees of freedom corresponding to the gauge singlet meson field of the magnetic theory. Indeed, the number of possible deformations of these D4-branes is

$$
N_{f}+2 \sum_{i=1}^{2 N_{f}-1}[i / 2]=\frac{2 N_{f}\left(2 N_{f}-1\right)}{2},
$$

which is equal to the number of independent components of the gauge-singlet meson field $\tilde{M}^{i j}=-\tilde{M}^{j i}$. 


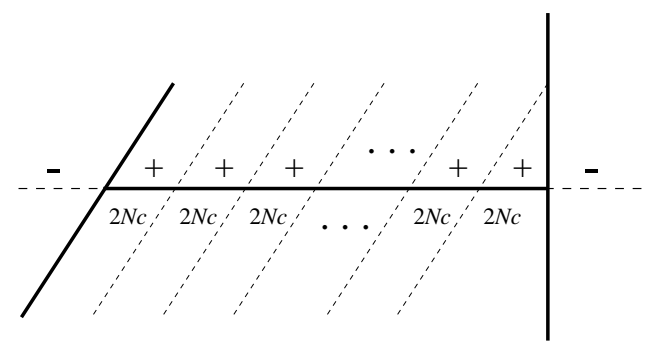

(e)

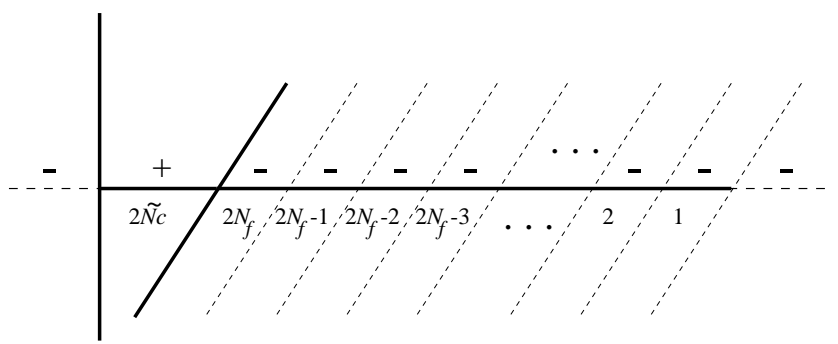

$(\mathrm{m})$

Figure 11: The Electric (e) and Magnetic (m) Limits (Symplectic Groups). "+" and "-" stand for $S p$-type and SO-type O4-planes respectively. The numbers below indicate the number of stretched D4-branes. We omit indicating the RR Wilson line (it is described in the main text). There are $2 N_{f}$ D6-branes in each.

As a summary, we depict in Figure $11(\mathrm{~m})$ the magnetic Type IIA limit.

\subsection{Dynamical Supersymmetry Breaking Model}

In [20] an $M$ theory fivebrane realization of Izawa-Yanagida-Intriligator-Thomas (IYIT) model of dynamical supersymmetry breaking was proposed. IYIT model is a supersymmetric $\operatorname{Sp}\left(N_{c}\right)$ QCD with $N_{f}=N_{c}+1$ flavors coupled to gauge singlet chiral multiplets $S_{i j}=-S_{j i}$ by the tree-level superpotential

$$
W_{\text {tree }}=\lambda S_{i j} Q^{i} Q^{j}
$$

where $\lambda$ is a coupling constant. Since the equation $\partial W / \partial S_{i j}=0$ is in conflict with the quantum modified constraint $\operatorname{Pf} Q^{i} Q^{j}=\Lambda^{4\left(N_{c}+1\right)}$ the model has no supersymmetric ground state. However, an analysis of the Kähler potential of the singlet field $S_{i j}$ at large values shows that there is a stable vacuum.

$W_{\text {tree }}$ in (4.5) looks like the tree-level superpotential of the magnetic theory considered above. Therefore, the goal is to find a fivebrane configuration that reduces to the Type IIA brane configuration as depicted in Figure $11(\mathrm{~m})$ with $\widetilde{N}_{c}$ replaced by $N_{c}$, which exhibits the correct behaviour of the gauge coupling constant at an energy much larger than $\Lambda$.

The proposal of [20] is essentially that the fivebrane configuration is given by that of the "electric dual", which is $\operatorname{Sp}\left(N_{e}\right) \mathrm{SQCD}, N_{e}=N_{f}-N_{c}-2$, whose dynamical scale

$\Lambda_{e}$ is related to the scale $\Lambda$ of the original model by $\Lambda^{3\left(N_{c}+1\right)-N_{f}} \Lambda_{e}^{3\left(N_{e}+1\right)-N_{f}}=\lambda^{-N_{f}}$. 
Actually, in the present case electric-magnetic duality does not hold since $N_{e}=\left(N_{c}+\right.$ 1) $-N_{c}-2=-1$. However, the equation (4.1) does make sense even if we put $N_{c}=-1$ and we can use it to define the holomorphic asymptotic condition of the fivebrane, thus defining a supersymmetric field theory in four-dimension. This proposal was shown to be consistent with the field theory results by observing an agreement of the moduli space of supersymmetric vacua of some perturbed models, including the model perturbed by a linear term in $S$,

$$
W_{\text {tree }}=\lambda S Q Q+m S
$$

which has supersymmetric vacua when $(m / \lambda)^{N_{f}}=\Lambda^{2 N_{f}}$. Here we provide a more elementary evidence by showing that the configurations have the required Type IIA limit.

The configuration of the model perturbed by the linear term $m S$ is in the elevendimensional space-time $\mathbf{R}^{4} \times M^{7} / \mathbf{Z}_{2}$ as in the case of $S p\left(N_{c}\right)$ SQCD but now the TaubNUT space is deformed as $y x=\left(v^{2}+m^{2}\right)^{N_{f}}$. The supersymmetric configuration of the model with $(m / \lambda)^{N_{f}}=\Lambda^{2 N_{f}}$ given in [20] is the configuration for the "electric dual" (4.1) where $N_{c}$ and $\Lambda$ there is replaced by $N_{e}=-1$ and $\Lambda_{e}$. Using the relation between $\Lambda_{e}$ and $\Lambda$, it is given by

$$
C^{\prime}\left\{\begin{array} { l } 
{ y = 1 } \\
{ v = 0 , }
\end{array} \quad C \left\{\begin{array}{l}
x=\lambda^{2\left(N_{c}+1\right)} \Lambda^{4\left(N_{c}+1\right)} \\
w=0
\end{array}\right.\right.
$$

(There is no $\mathrm{CP}^{1}$ component of the fivebrane.) The component $C$ intersects with the $\mathbf{Z}_{2}$ fixed plane $v=w=0$ at $x=\lambda^{2 N_{f}} \Lambda^{4 N_{f}}$ while the other component $C^{\prime}$ intersects at $x=m^{2 N_{f}}$. Therefore, the configuration is consistent only if $(m / \lambda)^{2 N_{f}}=\Lambda^{4 N_{f}}$, which is (almost) the same as the condition for unbroken supersymmetry in field theory. ${ }^{1}$

As before, we consider taking the limit $\Lambda \ll E$ for a fixed energy scale $E$ and look at the configuration (4.7) with respect to some fixed length scales in the $v$ and $w$ direction. As in the magnetic limit of SQCD, the component $C$ is sent to the left and wraps on the $\mathrm{CP}^{1}$ cycles as depicted in Figure 12. In the limit $R \ll \ell_{11}$, this reduces to a Type IIA brane configuration that obviously corresponds to IYIT model perturbed by a (very small but finite) linear term in $S$. If we force the pairs of D6-branes to approach each other at the orientifold plane, and then separate them in the $x^{6}$ direction, we will get the Type IIA configuration that looks like Figure $11(\mathrm{~m})$ with $\widetilde{N}_{c}$ replaced by $N_{c}$. Note that the curve is bent and is no longer holomorphic, and therefore it has some non-zero energy density.

\footnotetext{
${ }^{1}$ Actually, the configuration (4.7) can be deformed to a single component curve given by $v w=\lambda \Lambda^{2} \sigma$ and $x=\lambda^{2\left(N_{c}+1\right)} \Lambda^{4\left(N_{c}+1\right)}$, corresponding to the one-dimensional modulus space in field theory where $\sigma$ corresponds to the eigenvalue of the singlet $S$. However, we may restrict our attention to the $\sigma=0$ case (4.7), since we are interested in the energy much larger than $\Lambda$ and $S$.
} 


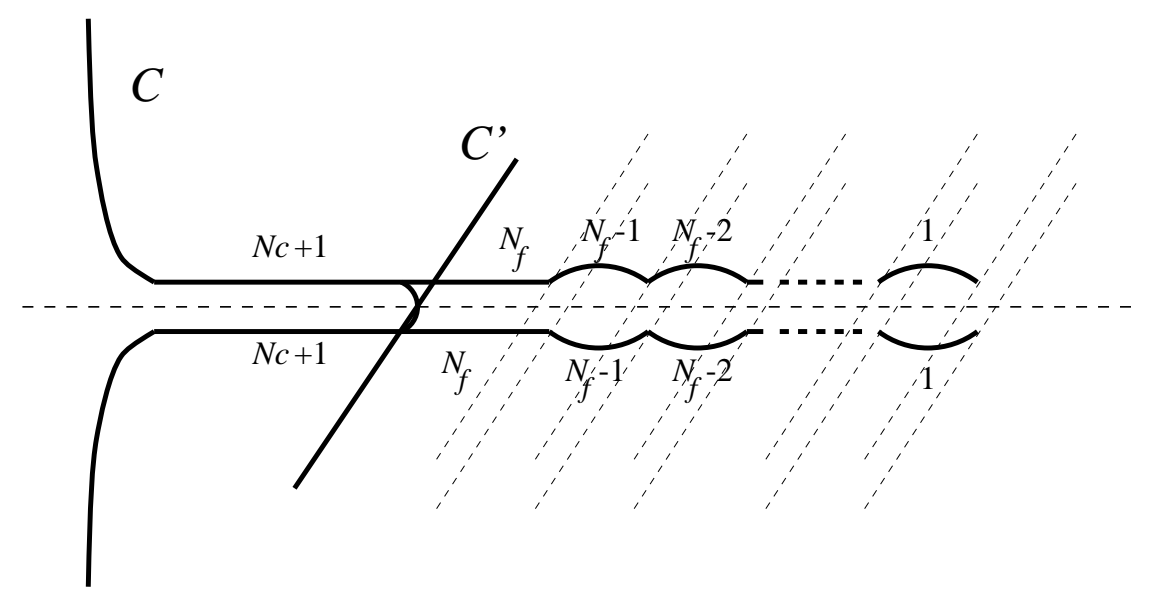

Figure 12: The $M$ Theory Configuration for the Perturbed IYIT Model in the Small $\Lambda$ Limit

Unlike in the magnetic dual of SQCD, there is no bending of the component $C^{\prime}$, and it appears that there is no natural way of setting the scale in the $w$ direction to measure the gauge coupling constant. However, we can use a knowledge about the perturbed models considered in [20]. Since the $w$ value of some characteristic point in such perturbed models is identified with the gauge singlet $S_{i j}$ which give a mass $\lambda S_{i j}$ to the quarks, the scale in the $w$ direction is set by $w \sim \lambda^{-1} E$. Then, the scale in the $v$ direction is set by $v \sim \lambda E^{2}$. Thus, the gauge coupling is measured as $\mathrm{e}^{-2 / g^{2}}=(\Lambda / E)^{4 N_{c}+4}$ which is the correct behaviour.

\section{Orthogonal Groups}

Here we consider the orthogonal gauge groups. The $M$ theory configuration for $N=1$ SQCD with even flavors can be obtained by rotating the $N=2$ configuration constructed in $[29,28]$. We find two Type IIA limits corrersponding to the elecetric and magnetic description of the theories.

\subsection{The Fivebrane Configuration}

The fivebrane configuration for $N=1 S O\left(N_{c}\right) \mathrm{SQCD}$ with $N_{f}$ flavors $\left(N_{f}\right.$ chiral multiplets in the vector representation) can be obtained by rotating the $N=2$ configuration in the standard way [3] if the flavor $N_{f}$ is even $\left(N_{f}\right.$ here means $2 N_{f}$ of ref.[28]). We first consider the range $N_{f} \geq N_{c}-1$. We present the result separately for even and odd $N_{c}$. 


\section{$N_{c}$ Even, $N_{f}$ Even}

The space-time in which the fivebrane is embedded is the $\mathbf{Z}_{2}$ quotient of the resolved $A_{N_{f}-1}$ Taub-NUT space $y x=v^{N_{f}}$ where $\mathbf{Z}_{2}$ acts as $y \rightarrow y, x \rightarrow x$ and $v \rightarrow-v$. The $\mathbf{Z}_{2}$ fixes the $\mathbf{C P}^{1}$ cycles $C_{2 i}$ point-wisely, but acts on $C_{2 i+1}$ as the $\pi$-rotation around the intersection points with $C_{2 i}$ and $C_{2 i+2}$.

The fivebrane consists of infinite components in $x^{7}=0$

$$
C^{\prime}\left\{\begin{array} { l } 
{ w ^ { 2 } y = \prod _ { i = 1 } ^ { N _ { c } / 2 } ( w ^ { 2 } - M _ { i } ^ { 2 } ) } \\
{ v = 0 }
\end{array} \quad C \left\{\begin{array}{l}
v^{2} x=\Lambda^{-\left(3\left(N_{c}-2\right)-N_{f}\right)} v^{N_{c}} \\
w=0
\end{array}\right.\right.
$$

and $\mathrm{CP}^{1}$ components wrapping the cycles $C_{i}$ with multiplicity $N_{c}$ for $i=1, \ldots, N_{f}-N_{c}+2$ and $N_{f}-i+2$ for $i=N_{f}-N_{c}+3, \ldots, N_{f}-1$. Note that $C$ includes a component described by $v^{2}=0$, the fivebrane wrapping twice on the $x$-axis. Also, the component $C^{\prime}$ at large $y$ looks almost like the fivebrane wrapping twice on the $y$-axis in $x^{7,8,9}=0$. These regions - large $\left|x^{6}\right|$ with $x^{4,5,7,8,9}=0$ - correspond in the Type IIA limit to the $\mathrm{O}^{+}$-plane (Sp-type, trivial RR Wilson line). The main component of $C, x=\Lambda^{-\left(3\left(N_{c}-2\right)-N_{f}\right)} v^{N_{c}-2}$ (which we denote again by $C$ ), intersects transversely with the $\mathrm{CP}^{1}$ cycle $C_{N_{f}-N_{c}+2}$. In addition, the fivebrane wraps odd number of times on the cycles $C_{2 i+1}$ (on which $\mathbf{Z}_{2}$ acts as the $\pi$-rotation) for $N_{f}-N_{c}+3 \leq 2 i+1 \leq N_{f}-1$. In order to avoid $t$ configurations, at least one pair of components wrapping $C_{2 i}$ must be at $x^{7,8,9}=0$ for each $2 i$ in $N_{f}-N_{c}+2 \leq 2 i<N_{f}-1$.

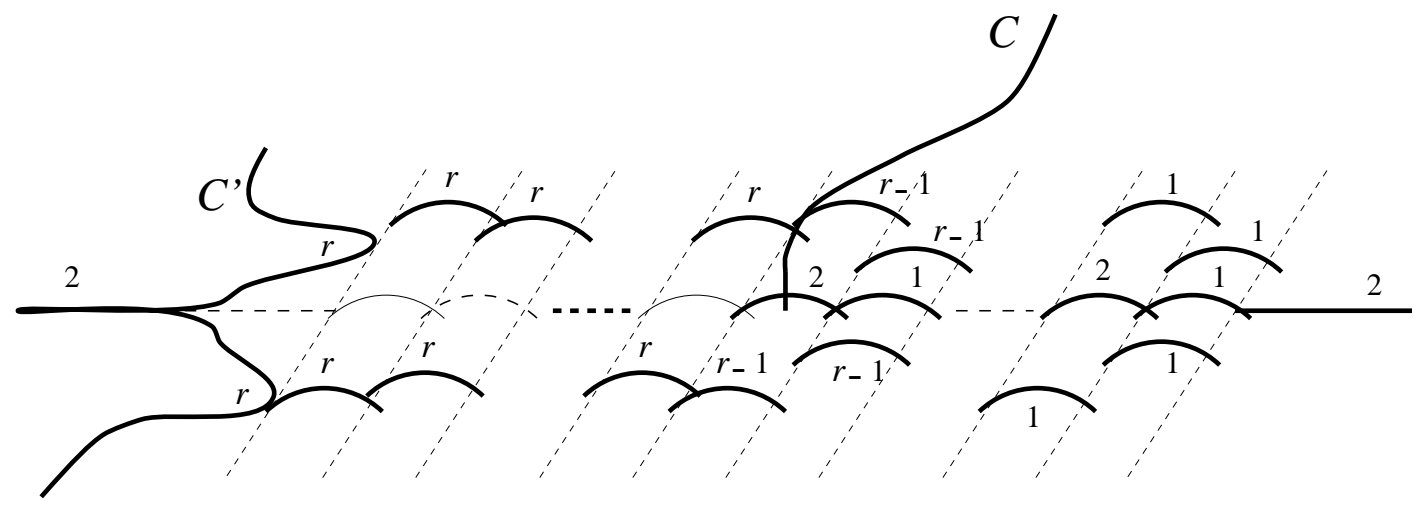

Figure 13: The $M$ Theory Configuration of $S O(2 r)$ SQCD.

There are $N_{f}$ sixbranes. The dashed horizontal lines and curves are point-wisely $\mathbf{Z}_{2}$ fixed while $\mathbf{Z}_{2}$ acts on the thin curves as the $\pi$-rotation.

The number of $\mathbf{Z}_{2}$ invariant deformations of the curve subject to this constraint counts 
as

$$
\frac{N_{c}}{2}+2\left[\left(N_{f}-N_{c}+1\right) \frac{N_{c}}{2}+2 \frac{\left(\frac{N_{c}}{2}-1\right) \frac{N_{c}}{2}}{2}\right]=N_{c} N_{f}-\frac{N_{c}\left(N_{c}-1\right)}{2}
$$

which agrees with the dimension of the moduli space of vacua. The parameters $\pm M_{i}$ are interpreted as the eigenvalues of $J_{i j} M^{j k}$ where $M^{i j}=Q_{a}^{i} Q_{a}^{j}$ is the vev of the meson matrix and $J_{i j}$ is the $S p\left(N_{f} / 2\right)$ invariant skew-symmetric form. Note that the eigenvalues of $J M$ come in pairs since ${ }^{t}(J M)=-J^{-1}(J M) J$.

$\underline{N_{c} \text { Odd, } N_{f} \text { Even }}$

The space-time in which the fivebrane is embedded is the $\mathbf{Z}_{2}$ quotient of the resolved $A_{N_{f}-1}$ Taub-NUT space $y x=v^{N_{f}}$ where $\mathbf{Z}_{2}$ acts as $y \rightarrow-y, x \rightarrow-x$ and $v \rightarrow-v$. The $\mathbf{Z}_{2}$ fixes the $\mathrm{CP}^{1}$ cycles $C_{2 i+1}$ point-wisely, but acts on $C_{2 i}$ as the $\pi$-rotation around the intersection points with $C_{2 i-1}$ and $C_{2 i+1}$.

The fivebrane consists of infinite components in $x^{7}=0$

$$
C^{\prime}\left\{\begin{array} { l } 
{ w y = \prod _ { i = 1 } ^ { [ N _ { c } / 2 ] } ( w ^ { 2 } - M _ { i } ^ { 2 } ) } \\
{ v = 0 }
\end{array} \quad C \left\{\begin{array}{l}
v x=\Lambda^{-\left(3\left(N_{c}-2\right)-N_{f}\right)} v^{N_{c}-1} \\
w=0
\end{array}\right.\right.
$$

and $\mathrm{CP}^{1}$ components wrapping the cycles $C_{i}$ with multiplicity $N_{c} \pm 1$ for $i=1, \ldots, N_{f}-$ $N_{c}+2$, and $N_{f}-i+2 \pm 1$ for $i=N_{f}-N_{c}+3, \ldots, N_{f}-1$ where upper case $(+1)$ is for odd $i$ and lower case $(-1)$ is for even $i$. Note that $C$ includes a component described by $v=0$, the fivebrane wrapping once on the $x$-axis. Also, the component $C^{\prime}$ at large $y$ looks almost like the fivebrane wrapping once on the $y$-axis. Noting that $\mathbf{Z}_{2}$ acts on the $y$ and $x$ axis at $x^{7,8,9}=0$ as the $\pi$-rotation around $y=0$ and $x=0$, we see that these regions — large $\left|x^{6}\right|$ with $x^{4,5,7,8,9}=0$ - correspond in the Type IIA limit to the $\widetilde{\mathrm{O}}^{+}$-plane $(S p$ type, non-trivial RR Wilson line). The main component of $C, x=\Lambda^{-\left(3\left(N_{c}-2\right)-N_{f}\right)} v^{N_{c}-2}$ (which we denote again by $C$ ), intersects transversely with the $\mathrm{CP}^{1}$ cycle $C_{N_{f}-N_{c}+2}$. In addition, the fivebrane wraps odd number of times on the cycles $C_{2 i}$ (on which $\mathbf{Z}_{2}$ acts as the $\pi$-rotation) for $N_{f}-N_{c}+3 \leq 2 i \leq N_{f}-1$ and also on the $x$-axis. In order to avoid a $t$-configuration, at least one pair of components wrapping $C_{2 i+1}$ must be at $x^{7,8,9}=0$ for each $2 i+1$ in $N_{f}-N_{c}+2 \leq 2 i+1 \leq N_{f}-1$.

The number of $\mathbf{Z}_{2}$ invariant deformations of the curve subject to this constraint counts as

$$
\frac{N_{c}-1}{2}+2\left[\frac{N_{f}-N_{c}+1}{2} \frac{N_{c}+1}{2}+\frac{N_{f}-N_{c}+1}{2} \frac{N_{c}-1}{2}+\frac{N_{c}-1}{2}+2 \frac{\frac{N_{c}-3}{2} \frac{N_{c}-1}{2}}{2}\right]=N_{c} N_{f}-\frac{N_{c}\left(N_{c}-1\right)}{2}
$$

which agrees with the dimension of the moduli space of vacua. The parameters $\pm M_{i}$ are interpreted as the eigenvalues of $J_{i j} M^{j k}$. 


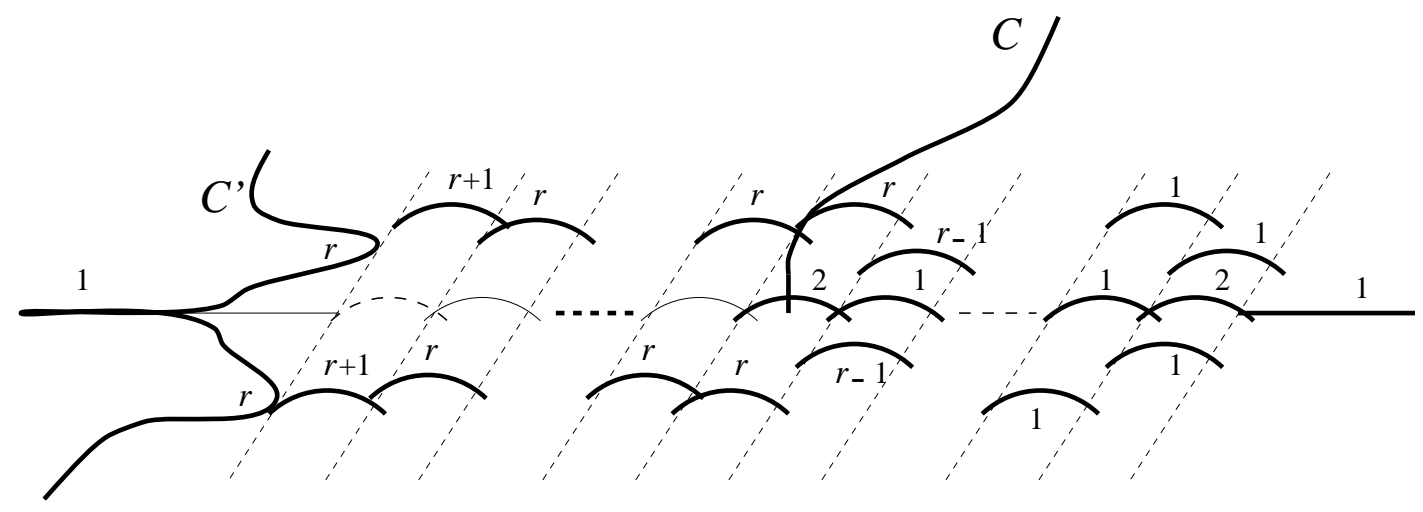

Figure 14: The $M$ Theory Configuration of $S O(2 r+1)$ SQCD.

There are $N_{f}$ sixbranes. The thin horizontal lines and curves are $\pi$-rotated by $\mathbf{Z}_{2}$ while the dashed curves are point-wisely $\mathbf{Z}_{2}$ fixed. Note the difference from $S O(2 r)$ case.

\subsection{The Electric and Magnetic Limits}

We examine the weakly coupled Type IIA limit of the $M$ theory configuration described above. In particular, we will find two limits which realizes the electric and magnetic theories of [33]. We explicitly write down the procedure for even $N_{c}$, but not for odd $N_{c}$ because the result is essentially the same and we only need a little more care.

\section{The Electric Limit}

Let us consider the limit $\Lambda^{3\left(N_{c}-2\right)-N_{f}} \ll E^{3\left(N_{c}-2\right)-N_{f}}$ for a fixed energy scale $E$ and look at the configuration with respect to the scale set by $v \sim E, w \sim E^{2}$. The main component of $C$ is sent to the right and wraps on the $\mathrm{CP}^{1}$ cycles $C_{N_{f}-N_{c}+3}, \ldots, C_{N_{f}-1}$ with multiplicities $1,2, \ldots, N_{c}-3$. It also wraps on the $x$-axis with mulitplicity $N_{c}-2$ and then blows up in the $v$-direction at $y(E)=\Lambda^{3\left(N_{c}-2\right)-N_{f}} E^{N_{f}-\left(N_{c}-2\right)}$. The component $C^{\prime}$ wraps on the $y$-axis and blows up in the $w$-direction at $y^{\prime}(E)=E^{2\left(N_{c}-2\right)}$. The two regions with large $v \gg E$ and large $w \gg E^{2}$ are interpreted as the NS and NS'5-branes in the Type IIA limit. Between them, the fivebrane wraps $N_{c}$ times on the eleventh direction. In the weakly coupled Type IIA limit, these are identified as the $N_{c}$ D4-branes stretched between the NS ${ }^{\prime}$ and the NS5-branes. The $\mathbf{Z}_{2}$ fixed cycle $C_{2 i}$ at $x^{7,8,9}=0$ is identified as $\mathrm{O} 4^{-}$-plane whereas the $\mathbf{Z}_{2}$ invariant but non-fixed cycle $C_{2 i+1}$ at $x^{7,8,9}=0$ is identified as the $\mathrm{O}^{0}$-plane. Namely, the region of O4-plane sandwiched between the two NS5-branes is basically of $S O$-type. Therefore the $N_{c}$ D4-branes yields $S O\left(N_{c}\right)$ gauge symmetry. 
The gauge coupling constant $g(E)$ at the energy $E$ can be read, as in the $S U\left(N_{c}\right)$ case, as

$$
\mathrm{e}^{-1 / g^{2}(E)}=\frac{y(E)}{y^{\prime}(E)}=\left(\frac{\Lambda}{E}\right)^{3\left(N_{c}-2\right)-N_{f}}
$$

As a summary, we depict in Figure 15 (e) the electric Type IIA limit. Note that the dimension of the moduli space can be counted also by applying the "s-rule" found in [28] to this configuration.

\section{The Magnetic Limit}

Let us next consider the opposite limit $\Lambda^{3\left(N_{c}-2\right)-N_{f}} \rightarrow \infty$. The component $C$ is sent to the left and wraps on the $\mathrm{CP}^{1}$ cycles $C_{1}, \ldots, C_{N_{f}-N_{c}+1}$ with multiplicities $N_{f}-N_{c}+$ $1, \ldots, 2,1$. It also wraps $N_{f}-N_{c}+2$ times on the $y$-axis and starts blowing up in the $v$-direction at some value of $y$. This value of $y$ is much larger than the value where the component $C^{\prime}$ starts blowing up in the $w$-direction with respect to some scale which will be specified momentarily. Between the two regions with large $v$ and $w$, the fivebrane wraps $\widetilde{N}_{c}=N_{f}-N_{c}+4$ times on the eleventh direction. In the Type IIA limit, it is interpreted as $\widetilde{N}_{c}$ degenerate D4-branes. Since these are on top of the $\mathrm{O} 4^{-}$-plane, we obtain $S O\left(\widetilde{N}_{c}\right)$ gauge symmetry. We can measure the gauge coupling at an energy $E$ by setting the corresponding length scales in the $v$ and $w$ directions. As in $S U\left(N_{c}\right)$ case, these length scales are set by $w \sim \mu E$ and $v \sim \mu^{-1} E^{2}$ where $\mu$ is a constant mass scale, and the $S O\left(\widetilde{N}_{c}\right)$ gauge coupling $\widetilde{g}(E)$ is measured as

$$
\mathrm{e}^{-1 / \widetilde{g}^{2}(E)}=\frac{\mu^{N_{f}} \Lambda^{-3\left(\widetilde{N}_{c}-2\right)+N_{f}}}{E^{3\left(\widetilde{N}_{c}-2\right)-N_{f}}} .
$$

This yields the standard relation $\Lambda^{3\left(\widetilde{N}_{c}-2\right)-N_{f}} \widetilde{\Lambda}^{3\left(\widetilde{N}_{c}-2\right)-N_{f}}=\mu^{N_{f}}$ between the dynamical scales of the electric and magnetic theories [26].

On the right of the region of $C^{\prime}$ with large $w \gg \mu E$ (which is identified as the $\mathrm{NS}^{\prime} 5$ brane), we have components of the fivebrane wrapping several times on the eleventh direction. The fivebrane wraps $N_{f}+2$-times in the region sandwiched between the NS'5brane and the left-most D6-brane, while it wraps $i+2$-times on the $\mathrm{CP}^{1}$ cycle $C_{N_{f}-i}$. In the weakly coupled Type IIA limit $R \ll \ell_{11}$, two of the fivebranes in the region sandwiched between the $\mathrm{NS}^{\prime} 5$-brane and the left-most D6-brane are interpreted as providing a charge +2 to the orientifold fixed plane (consituting the $\mathrm{O} 4^{+}$-plane). Also, a pair of fivebranes wrapping each of the $\mathbf{Z}_{2}$ fixed cycles (namely, the $\mathbf{C P}^{1}$ cycles $C_{2 i}$ and the $x$-axis at $\left.x^{7,8,9}=0\right)$ is interpreted as the $\mathrm{O}^{+}$-plane. Similarly, a single fivebrane wrapped on 
each of the $\mathbf{Z}_{2}$ invariant cycles $C_{2 i+1}$ at $x^{7,8,9}=0$ (on which $\mathbf{Z}_{2}$ acts as the $\pi$-rotation) is interpreted as the $\widetilde{\mathrm{O}} 4^{+}$-plane. The rest of the fivebrane wraps $N_{f}$-times in the region sandwiched between the $\mathrm{NS}^{\prime} 5$-brane and the left-most D6-brane, while it wraps $2[(i+1) / 2]$ times on the cycles $C_{N_{f}-i}$. These are interpreted as the D4-branes which represent the degrees of freedom corresponding to the gauge singlet meson field of the magnetic theory. Indeed, the number of possible deformations of these D4-branes is

$$
\frac{N_{f}}{2}+2 \sum_{i=1}^{N_{f}-1}[(i+1) / 2]=\frac{N_{f}\left(N_{f}+1\right)}{2},
$$

which is equal to the number of independent components of the singlet meson field $\tilde{M}^{i j}=$ $\tilde{M}^{j i}$.

As a summary, we depict in Figure $15(\mathrm{~m})$ the magnetic Type IIA limit. The O4planes that extend to the left and right infinity are $\mathrm{O}^{+}{ }^{+}$-plane. $\mathrm{O}^{+}$and $\widetilde{\mathrm{O}}^{+}$appears alternately since D6-brane is a unit magnetic monopole for the RR $U(1)$ gauge field.

\section{$\operatorname{Odd} N_{c}$}

We can consider the Type IIA limit for odd $N_{c}$ in a similar way. It turns out that the result is the same: we can find the electric and magnetic limits which are given exactly as in Figure $15(\mathrm{e})$ and $(\mathrm{m})$, where $N_{c}$ and $\widetilde{N}_{c}$ are odd now. The O4-planes that extend to the left and right infinity are $\widetilde{\mathrm{O}}^{+}$-plane in the present case. In the magnetic configuration, $\widetilde{\mathrm{O}}^{+}$and $\mathrm{O}^{+}$again appears alternately as we cross D6-branes.

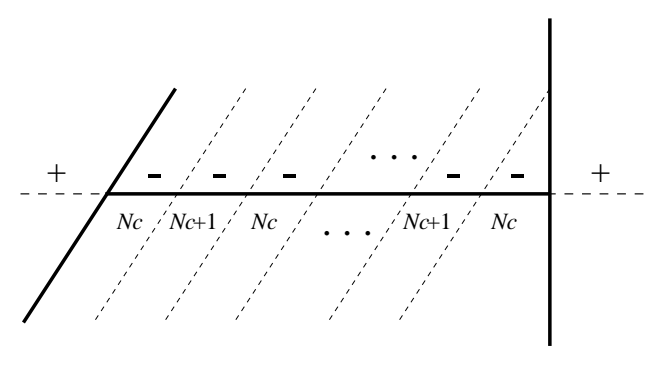

(e)

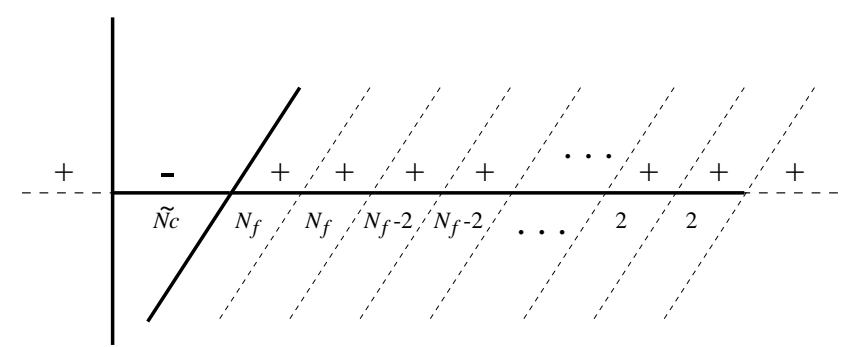

$(\mathrm{m})$

Figure 15: The Electric (e) and Magnetic (m) Type IIA Limits (Orthogonal Gauge Groups) There are $N_{f}$ D6-branes in each. 


\section{3 $N_{f}=N_{c}-4, N_{c}-3$ : Confining Phase}

In the rest of the paper, we consider the remaining cases $0<N_{f}<N_{c}-2$. We again restrict our attention to the case where $N_{f}$ is even.

$\underline{N_{f}<N_{c}-4}$

For $N_{f}<N_{c}-4$, there is no fivebrane configuration that lifts the electric Type IIA configuration (Figure $15(\mathrm{e})$ ), as can be seen as follows. The asymptotics at large $v$ and large $x$ requires that the fivebrane has a component $C$ described by (5.1) or (5.3). However, the equation for $C$ implies $y v^{N_{c}-2-N_{f}}=\Lambda^{3\left(N_{c}-1\right)-N_{f}}$ where the power of $v$ is at least $4\left(N_{c}\right.$ even) or $3\left(N_{c}\right.$ odd $)$ when $N_{f}<N_{c}-4$. Since $v^{2}=0\left(N_{c}\right.$ even $)$ or $v=0\left(N_{c}\right.$ odd) at large $y$ represents the $S p$-type O4-plane, this shows that there are extra D4-branes on the left of the NS'5-brane, which is not the property of the Type IIA configuration. The absence of $M$ theory configuration corresponds to the absence of (supersymmetric) stable vacua due to the generation of Affleck-Dine-Seiberg superpotential.

$\underline{N_{f}=N_{c}-4}\left(N_{c}\right.$ Even $)$

The fivebrane consists of infinite components at $x^{7}=0$

$$
C^{\prime}\left\{\begin{array} { l } 
{ y = w ^ { 2 } \prod _ { i = 1 } ^ { N _ { f } / 2 } ( w ^ { 2 } - M _ { i } ^ { 2 } ) } \\
{ v = 0 }
\end{array} \quad C \left\{\begin{array}{l}
v^{2} x=\Lambda^{-\left(3\left(N_{c}-2\right)-N_{f}\right)} v^{N_{c}} \\
w=0,
\end{array}\right.\right.
$$

and $\mathrm{CP}^{1}$ components wrapping $C_{1}, \ldots, C_{N_{f}-1}$ with multiplicity $N_{c}-3, N_{c}-4, \ldots, 4,3$. Again $C$ includes a component wrapping twice on the $x$-axis. The strong coupling limit $\Lambda \rightarrow \infty$ of this configuration leads to a configuration which looks like the magnetic configuration of Figure $15(\mathrm{~m})$ extrapolated to $N_{f}=N_{c}-4$, namely, $\widetilde{N}_{c}=0$. We see that there is no gauge symmetry in this configuration but there are degrees of freedom as many as $N_{f}\left(N_{f}+1\right) / 2$ chiral superfields. This is consistent with the field theory knowledge [33] that the theory confines and becomes a theory of composite meson field $M^{i j}=Q^{i} Q^{j}$.

$\underline{N_{f}=N_{c}-3}\left(N_{c}\right.$ Odd $)$

The fivebrane consists of infinite components at $x^{7}=0$

$$
C^{\prime}\left\{\begin{array} { l } 
{ y = w \prod _ { i = 1 } ^ { N _ { f } / 2 } ( w ^ { 2 } - M _ { i } ^ { 2 } ) } \\
{ v = 0 }
\end{array} \quad C \left\{\begin{array}{l}
v x=\Lambda^{-\left(3\left(N_{c}-2\right)-N_{f}\right)} v^{N_{c}-1} \\
w=0
\end{array}\right.\right.
$$

and $\mathrm{CP}^{1}$ components wrapping $C_{i}$ with multiplicity $N_{c}-1-i \pm 1$ where +1 is for odd $i$ and -1 is for even $i$. $C$ includes a component wrapping once over the $x$-axis. The strong coupling limit $\Lambda \rightarrow \infty$ of this configuration leads to a configuration which looks like the magnetic configuration of Figure $15(\mathrm{~m})$ extrapolated to $N_{f}=N_{c}-3$, namely, 
$\widetilde{N}_{c}=1$. We see that there is no gauge symmetry in this configuration but there are degrees of freedom as many as $N_{f}\left(N_{f}+1\right) / 2$ chiral superfields. Also, there are $N_{f}$ modes associated with the open string stretched between the single D4-brane on top of O4-plane and the $N_{f}$ D4-branes ending on the NS'5-brane. The mass of these modes are given by $M_{i}$ which are identified as the meson eigenvalues. This is consistent with the field theory knowledge [33] that the theory confines and becomes a theory of composite meson field $M^{i j}$ and composite $N_{f}$ fields $q_{i}$ (glueballs or exotics) coupled via a superpotential of the form $M^{i j} q_{i} q_{j}$.

\section{4 $N_{f}=N_{c}-2$ : Coulomb Phase}

The fivebrane for the case $N_{f}=N_{c}-2\left(N_{c}\right.$ even $)$ consists of infinite components at $x^{7}=0$

$$
C^{\prime}\left\{\begin{array} { l } 
{ w ^ { 2 } y = w ^ { 2 } \prod _ { i = 1 } ^ { N _ { f } / 2 } ( w ^ { 2 } - M _ { i } ^ { 2 } ) } \\
{ v = 0 }
\end{array} \quad C \left\{\begin{array}{l}
v^{2} x=\Lambda^{-2\left(N_{c}-2\right)} v^{N_{c}} \\
w=0,
\end{array}\right.\right.
$$

and $\mathrm{CP}^{1}$ components wrapping $C_{1}, \ldots, C_{N_{f}-1}$ with multiplicity $N_{c}-1, N_{c}-2, \ldots, 4,3$. $C$ includes a component wrapping twice on the $x$-axis, and $C^{\prime}$ includes a component wrapping twice on the $y$-axis. The main component of $C$ is decribed by $y=\Lambda^{2\left(N_{c}-2\right)}$ and intersects transversely with the $y$-axis. The main component of $C^{\prime}$ also intersects transversely with the $y$-axis at $y=\prod_{i}\left(-M_{i}^{2}\right)$. The $y$-axis is a $\mathbf{Z}_{2}$ fixed plane but the intersection with a fivebrane is allowed because it is screened by a pair of fivebranes (see [28]).

In the weak coupling region, all $M_{i} \gg \Lambda^{2}, C^{\prime}$ is on the left of $C$ in a neighborhood of the $y$-axis. In the strong coupling region, all $M_{i} \ll \Lambda^{2}, C$ is on the left of $C^{\prime}$ in a neighborhood of the $y$-axis, just as in the magnetic configuration (Figure $15(\mathrm{~m})$ ). For both cases, in the weakly coupled Type IIA limit, the region of the $y$-axis sandwiched between $C$ and $C^{\prime}$ is identified as $\mathrm{O}^{-}$-plane with a pair of D4-branes. Namely, we see the $S O(2)$ gauge symmetry in both weak and strong coupling regions. In the strong coupling region, there are $N_{f}$ electrons transforming in the vector representation of $S O(2)$ with mass given by $M_{i}$, as the megnetic configuration shows. These two $S O(2)$ gauge theories, electric and magnetic $S O(2) \mathrm{s}$, are continuously connected by changing $M_{i}$ 's because the intersection point $y=\prod_{i}\left(-M_{i}^{2}\right)$ of $C^{\prime}$ can go around the intersection point $y=\Lambda^{2\left(N_{c}-2\right)}$ of $C$. Something interesting happens when these intersection points collide, $\prod_{i=1}^{N_{f} / 2}\left(-M_{i}^{2}\right)=\Lambda^{2\left(N_{c}-2\right)}$.

These are qualitatively the same as what we know for field theory. However, we know from field theory considerations more than just the qualitative features. For example, 
the exact effective $S O(2)$ gauge coupling $g_{\text {eff }}$ has been determined by finding the SeibergWitten curve [33]. In the brane picture, it is not obvious how to compute it. We can only tell that $1 / g^{2} \sim \log \left(\prod_{i} M_{i}^{2} / \Lambda^{2\left(N_{c}-2\right)}\right)$ in the weak coupling region, which is roughly correct but not exact. The curve is completely degenerate and there is no way to make it a smooth genus one Riemann surface without changing the asymptotic behaviour, as can be seen by noting the existence of a meromorphis function with a single simple pole. The effective gauge coupling constant should be determined in a different way than using the Seiberg-Witten type curve. Also, the field theory argument [33] shows that there is a dyon which becomes massless at the colliding point $\prod_{i=1}^{N_{f} / 2}\left(-M_{i}^{2}\right)=\Lambda^{2\left(N_{c}-2\right)}$. It is not clear whether such a dyon state exists in the brane picture. It is challenging to solve these problems. It might be related to something important in $M$ theory on $\mathbf{R}^{5} / \mathbf{Z}_{2}$ orbifold.

\section{Acknowledgement}

I would like to thank C. Csaki, J. de Boer, A. Giveon, H. Murayama, H. Ooguri, Y. Oz, S.-J. Rey, M. Schmaltz, M. Strassler, R. Sundrum and C. Vafa for discussions. I thank Institute for Theoretical Physics at Santa Barbara for hospitality.

This research is supported in part by NSF grant PHY-95-14797 and DOE grant DEAC03-76SF00098, and also by NSF grant PHY94-07194. 


\section{Appendix}

\section{A Vacuum Structure of $N=2$ SQCD Perturbed to $N=1$}

In the previous work [3], $N=2 S U\left(N_{c}\right)$ SQCD with $N_{f}$ flavors perturbed by a mass term for the adjoint chiral multiplet was studied both from field theory method and from brane description. For $N_{f}>N_{c}+1$, there was a gap between the results of the two methods concerning the vacua with meson matrix of rank $<N_{f}$. In this appendix, we correct an error in the field theory treatment of [3] and present a better argument showing that the brane result was correct.

By integrating out the adjoint chiral multiplet of mass $\mu_{\mathrm{a}}$, the system can be considered as $N=1$ SQCD perturbed by the chiral symmetry breaking superpotential

$$
\Delta W=\frac{1}{2 \mu_{\mathrm{a}}}\left[\operatorname{Tr}(\widetilde{Q} Q \widetilde{Q} Q)-\frac{1}{N_{c}}(\operatorname{Tr} \widetilde{Q} Q)^{2}\right]
$$

at the cut-off scale $\mu_{\mathrm{a}}$. As far as the gauge group $S U\left(N_{c}\right)$ is broken at high energy by

a large vev of $Q$ or $\widetilde{Q}$, one can use this to analyze the space of vacua. However, once $Q$ and $\widetilde{Q}$ become smaller than $\Lambda$ (as it turns out, all branch of the moduli space is in or connected to this region), the gauge coupling becomes strong and it is no longer valid to use the elementary field. For $N_{c}+1<N_{f} \leq 3 N_{c} / 2$, it is appropriate to use the magnetic description of the theory which becomes weakly coupled at low energy. Even for $3 N_{c} / 2<N_{f}<2 N_{c}$, although the theory without perturbation (A.1) flows to a non-trivial fixed point, it turns out that the moduli space of the theory with (A.1) is in or connected to a region where we can find an appropriate description starting from the magnetic theory. Thus, we consider the $S U\left(\widetilde{N}_{c}\right)$ gauge theory with $N_{f}$ quarks $q_{i}, \widetilde{q}^{i}$ (magnetic quarks) and a gauge singlet meson $M\left(N_{f} \times N_{f}\right.$ matrix) which are coupled by tree-level superpotential

$$
W_{\text {tree }}=\frac{1}{\mu} M q \widetilde{q}+\frac{1}{2 \mu_{\mathrm{a}}}\left[\operatorname{Tr}\left(M^{2}\right)-\frac{1}{N_{c}}(\operatorname{Tr} M)^{2}\right] .
$$

The error of the treatment in [3] was that we assumed following [11] that there exists an exact superpotential that applies for any rank of $M\left(=\right.$ the sum of $W_{\text {tree }}$ and an AffleckDine-Seiberg type potential). However, this potential is not holomorphic at $\operatorname{det} M=0$ and the analysis is not valid for $\operatorname{rank} M<N_{f}$. In fact, the analysis should depend on the rank of $M$ since the low energy theory depends drastically on the number of massless quarks which is determined by $\operatorname{rank} M$.

If the rank of $M$ is $N_{f}$, all the magnetic quarks are massive and the low energy theory is the pure $S U\left(\widetilde{N}_{c}\right)$ super-Yang-Mills theory with the dynamical scale $\Lambda_{L}$ given 
by $\Lambda_{L}^{3 \widetilde{N}_{c}}=\operatorname{det}(M / \mu) \widetilde{\Lambda}^{3 \widetilde{N}_{c}-N_{f}}$. Thus, the theory in this branch is described by the exact superpotential

$$
W_{\text {eff }}=\widetilde{N}_{c} \Lambda_{L}^{3}+\frac{1}{2 \mu_{\mathrm{a}}}\left[\operatorname{Tr}\left(M^{2}\right)-\frac{1}{N_{c}}(\operatorname{Tr} M)^{2}\right] .
$$

Extremizing $W_{\text {eff }}$, we obtain the maximal rank solutions which were obtained in [3] both from field theory and from brane analysis. Indeed, the solution is of order $M \sim$ $\left(\Lambda^{3 N_{c}-N_{f}} / \mu_{\mathrm{a}}^{N_{f}-N_{c}}\right)^{1 /\left(2 N_{c}-N_{f}\right)} \ll \Lambda^{2}$ for $\mu_{\mathrm{a}} \gg \Lambda$, and is inaccessible from the electric description.

If the rank $\ell$ of $M$ is smaller $\ell<N_{f}, N_{f}^{\prime}=N_{f}-\ell$ of the quarks remain massless. Let us decompose the meson matrix into blocks of size $\ell$ and $N_{f}^{\prime}$ as

$$
M=\left(\begin{array}{cc}
M_{H} & M_{12} \\
M_{21} & M_{L}
\end{array}\right)
$$

where $\operatorname{rank}\left\langle M_{H}\right\rangle=\ell,\left\langle M_{12}\right\rangle=\left\langle M_{21}\right\rangle=\left\langle M_{L}\right\rangle=0$. By integrating out the $\ell$ massive quarks with mass matrix $M_{H} / \mu$, we obtain the low energy theory which is $S U\left(\widetilde{N}_{c}\right)$ SQCD with $N_{f}^{\prime}=N_{f}-\ell$ quarks $q_{L i}, \widetilde{q}_{L}^{i}$ with the dynamical scale $\Lambda_{L}$ and the tree-level superpotential $W_{L}$ given by

$$
\begin{gathered}
\Lambda_{L}^{3 \widetilde{N}_{c}-N_{f}^{\prime}}=\operatorname{det}\left(\frac{M_{H}}{\mu}\right) \widetilde{\Lambda}^{3 \widetilde{N}_{c}-N_{f}} \\
W_{L}=\frac{1}{\mu} M_{L} q_{L} \widetilde{q}_{L}+\frac{1}{2 \mu_{\mathrm{a}}}\left[\operatorname{Tr}\left(M^{2}\right)-\frac{1}{N_{c}}(\operatorname{Tr} M)^{2}\right] .
\end{gathered}
$$

For $N_{c}<\ell<N_{f}$, since the flavor $N_{f}^{\prime}$ of the low energy theory is smaller than the color $\widetilde{N}_{c}$, Affleck-Dine-Seiberg superpotential is generated

$$
W_{e f f}=W_{L}+\left(\widetilde{N}_{c}-N_{f}^{\prime}\right)\left(\frac{\Lambda_{L}^{3 \widetilde{N}_{c}-N_{f}^{\prime}}}{\operatorname{det} q_{L} \widetilde{q}_{L}}\right)^{1 /\left(\widetilde{N}_{c}-N_{f}^{\prime}\right)}
$$

It is easy to see that there is no solution to $\partial W_{\text {eff }}=0$ which satisfies $M_{L}=0$. Hence there is no vacuum with $N_{c}<\operatorname{rank} M<N_{f}$.

For $\ell=N_{c}$, the low energy theory is $S U\left(\widetilde{N}_{c}\right)$ with flavor $N_{f}^{\prime}=\widetilde{N}_{c}$. At energies far below $\Lambda_{L}$, this theory is described by composite mesons $N_{L}=q_{L} \widetilde{q}_{L}$ and baryons $b_{L}=\operatorname{det} q_{L}$, $\widetilde{b}_{L}=\operatorname{det} \widetilde{q}_{L}$ which are subject to the quantum modified constraint $\operatorname{det} N_{L}-b_{L} \widetilde{b}_{L}=\Lambda_{L}^{2 \widetilde{N}_{c}}$. Thus, the effective superpotential is

$$
W_{\text {eff }}=\frac{1}{\mu} \operatorname{tr}\left(M_{L} N_{L}\right)+\frac{1}{2 \mu_{\mathrm{a}}}\left[\operatorname{Tr}\left(M^{2}\right)-\frac{1}{N_{c}}(\operatorname{Tr} M)^{2}\right]+X\left(\operatorname{det} N_{L}-b_{L} \widetilde{b}_{L}-\Lambda_{L}^{2 \widetilde{N}_{c}}\right) .
$$


The equation $\partial W_{\text {eff }}=0$ together with $M_{L}=M_{12}=M_{21}=0$ requires

$$
\begin{aligned}
& N_{L}=\frac{\mu}{N_{c} \mu_{\mathrm{a}}} \operatorname{Tr} M_{H} \mathbf{1}_{\widetilde{N}_{c}} \\
& M_{H}-\frac{1}{N_{c}} \operatorname{Tr} M_{H}=0 \\
& \operatorname{det} N_{L}-b_{L} \widetilde{b}_{L}=\Lambda_{L}^{2 \widetilde{N}_{c}}
\end{aligned}
$$

The first and second equations implies $M_{H}=m \mathbf{1}_{N_{c}}$ and $N_{L}=\left(\mu m / \mu_{\mathrm{a}}\right) \mathbf{1}_{\widetilde{N}_{c}}$ for some $m$. Then the quantum modified constraint (A.11) of the low energy theory yields

$$
\left(m / \mu_{\mathrm{a}}\right)^{\widetilde{N}_{c}}-b_{L} \widetilde{b}_{L} / \mu^{\widetilde{N}_{c}}=\Lambda_{L}^{2 \widetilde{N}_{c}} / \mu^{\widetilde{N}_{c}} .
$$

By using the relation between the electric and magnetic baryon operators $b_{L}=b_{N_{c}+1, \ldots, N_{f}}=$ $C^{-1} B^{1, \ldots, N_{c}}$ and $\widetilde{b}_{L}=\widetilde{b}^{N_{c}+1, \ldots, N_{f}}=C^{-1} \widetilde{B}_{1, \ldots, N_{c}}, C=\left(-(-\mu)^{-\widetilde{N}_{c}} \Lambda^{3 N_{c}-N_{f}}\right)^{1 / 2}$, together with the scale matching equation (A.5), we obtain

$$
m^{N_{c}}+B \widetilde{B}=(-1)^{N_{f}-N_{c}}\left(\frac{m}{\mu_{\mathrm{a}}}\right)^{N_{f}-N_{c}} \Lambda^{3 N_{c}-N_{f}} .
$$

This is nothing but the relation obtained in [3] from the brane description of the theory.

For $\ell=N_{c}-1$, the low energy theory is $S U\left(\widetilde{N}_{c}\right)$ SQCD with $N_{f}^{\prime}=\widetilde{N}_{c}+1$ flavors. At energies far below $\Lambda_{L}$, this theory is described by composite mesons $N_{L}$ and baryons $b_{L}, \widetilde{b}_{L}$ and the superpotential is given by

$$
W_{\text {eff }}=\frac{1}{\mu} \operatorname{tr}\left(M_{L} N_{L}\right)+\frac{1}{2 \mu_{\mathrm{a}}}\left[\operatorname{Tr}\left(M^{2}\right)-\frac{1}{N_{c}}(\operatorname{Tr} M)^{2}\right]+\frac{1}{\Lambda_{L}^{2 \widetilde{N}_{c}-1}}\left(\operatorname{det} N_{L}-b_{L} N_{L} \widetilde{b}_{L}\right) .
$$

It is easy to see that there is no solution to $\partial W_{\text {eff }}=0$ with $M_{L}=0$ and $\operatorname{det} M_{H} \neq 0$. Therefore, there is no vacuum with $\operatorname{rank} M=N_{c}-1$.

For $\ell<N_{c}-1$, the low energy theory is $S U\left(\widetilde{N}_{c}\right)$ SQCD with $N_{f}^{\prime}>\widetilde{N}_{c}$. It is known that this theory exhibits no generation of superpotential, and the classical analysis for determining vacua is valid as far as the moduli space extends to a region where the gauge coupling stops running at high energy. The vacuum equation $\partial W_{L}=0$ is satisfied when $M_{H}=0$ (which implies $\ell=0$ ) and $q_{i} \widetilde{q}^{j}=0$, and in fact either $q_{i}$ 's or $\widetilde{q}^{j}$ 's can have large expectation values of rank $\widetilde{N}_{c}$. From the equation $q_{i} \widetilde{q}^{j}=0$, we see that $b_{i_{1}, \ldots, i \widetilde{N}_{c}} \widetilde{b}^{j_{1}, \ldots, j}{\widetilde{N_{c}}}_{c}=0$. Thus, this branch is given by $M=0, B^{i_{1}, \ldots, i_{N_{c}} \widetilde{B}_{j_{1}, \ldots, j_{N_{c}}}}=0$. This is actually the $m \rightarrow 0$ limit of the branch already obtained above (A.13).

One can also show that the brane tells the dimension of the moduli space correctly. For the maximal rank solutions, we have already seen in [3] that both field theory and 
brane analysis gives complex dimension $2 r\left(N_{f}-r\right)$. For the other branch, the brane consists of $N_{c}\left(N_{f}-N_{c}\right) \mathrm{CP}^{1}$ components and two factorized infinite components. If we include the relative motion of the infinite components as one of its moduli (which is still an unanswered problem), the complex dimension is $2\left(N_{f} N_{c}-N_{c}^{2}+1\right)$. On the field theory side, as we have seen above, the solution can be made to the following form by the complexified flavor symmetry $G L\left(N_{f}, \mathbf{C}\right)$ :

$$
\begin{aligned}
& M=\left(\begin{array}{cc}
m \mathbf{1}_{N_{c}} & \\
& \mathbf{0}_{N_{f}-N_{c}}
\end{array}\right) \\
& B^{1, \ldots, N_{c}}=B, \quad \text { other components }=0 \\
& \widetilde{B}_{1, \ldots, N_{c}}=\widetilde{B}, \quad \text { other components }=0
\end{aligned}
$$

where $m, B$ and $\widetilde{B}$ obey (A.13). The unbroken subgroup of the flavor symmetry $G L\left(N_{f}, \mathbf{C}\right)$ at this solution is $S L\left(N_{c}, \mathbf{C}\right) \times G L\left(N_{f}-N_{c}, \mathbf{C}\right)$. Since the flavor invariants are parametrized by one complex modulus due to the constraint (A.13), the complex dimension of the moduli space is $1+N_{f}^{2}-\left(N_{c}^{2}-1+\left(N_{f}-N_{c}\right)^{2}\right)=2\left(N_{f} N_{c}-N_{c}^{2}+1\right)$ which agrees with the result of the brane analysis. 


\section{References}

[1] A. Giveon and D. Kutasov, Brane Dynamics and Gauge Theory, hep-th/9802067.

[2] E. Witten, "Solutions Of Four-Dimensional Field Theories Via M Theory," hep-th/9703166, Nucl.Phys. B500 (1997) 3-42.

[3] K. Hori, H. Ooguri and Y. Oz, "Strong Coupling Dynamics of Four-Dimensional N=1 Gauge Theories from M Theory Fivebrane," hep-th/9706082, Adv. in Theor. and Math. Phys. 1 (1997) 1.

[4] E. Witten, "Branes And The Dynamics Of QCD," hep-th/9706109.

[5] A. Brandhuber, N. Itzhaki, V. Kaplunovsky, J. Sonnenschein and S. Yankielowicz, "Comments on the M Theory Approach to N=1 SQCD and Brane Dynamics," hep-th/9706127, Phys.Lett. B410 (1997) 27-35.

[6] J. de Boer, K. Hori, H. Ooguri and Y. Oz, "Kahler Potential and Higher Derivative Terms from M Theory Fivebrane," hep-th/9711143.

[7] S. Elitzur, A. Giveon, D. Kutasov, "Branes and N=1 Duality in String Theory," hepth/9702014, Phys.Lett. B400 (1997) 269-274.

[8] N. Seiberg, "Electric-Magnetic Duality in Supersymmetric Non-Abelian Gauge Theories," hep-th/9411149, Nucl.Phys. B435 (1995) 129-146.

[9] H. Ooguri and C. Vafa, "Geometry of N=1 Dualities in Four Dimensions", hep-th/9702180, Nucl.Phys. B500 (1997) 62-74.

[10] N. Evans, C. Johnson and A. Shapere, " Orientifolds, Branes, and Duality of 4D Gauge Theories", hep-th/9703210, Nucl.Phys. B505 (1997) 251-271.

[11] S. Elitzur, A. Giveon, D. Kutasov, E. Rabinovici and A. Schwimmer, "Brane Dynamics and N=1 Supersymmetric Gauge Theory," hep-th/9704104, Nucl.Phys. B505 (1997) 202-250.

[12] M. Schmaltz and R. Sundrum, "N = 1 Field Theory Duality from M-theory," hepth/9708015.

[13] C. Csaki and W. Skiba, "Duality in Sp and SO Gauge Groups from M Theory," hepth/9708082.

[14] C. Csaki, M. Schmaltz, W. Skiba and J. Terning, "Gauge Theories with Tensors from Branes and Orientifolds", hep-th/9801207.

[15] N. Evans, "Duality in Non-Supersymmetric MQCD", hep-th/9804097.

[16] S. Sugimoto, "Comments on Duality in MQCD", hep-th/9804114.

[17] T. Nakatsu, K. Ohta, T. Yokono and Y. Yoshida, "A Proof of Brane Creation via M theory," hep-th/9711117. 
[18] Y. Yoshida, "Geometrical Analysis of Brane Creation via M-Theory," hep-th/9711177.

[19] T. Nakatsu, K. Ohta and T. Yokono, "On the Baryonic Branch Root of N=2 MQCD," hep-th/9712029.

[20] J. de Boer, K. Hori, H. Ooguri and Y. Oz, "Branes and Dynamical Supersymmetry Breaking", hep-th/9801060.

[21] V.A. Novikov, M.A. Shifman, A.I. Vainshtein and V.I. Zakharov, "Exact GellMann-Low Function of Supersymmetric Yang-Mills Theories from Instanton Calculus," Nucl.Phys.B229 (1983) 381; "The Beta Function In Supersymmetric Gauge Theories. Instanton versus Traditional Approach," Phys.Lett.166B (1986) 329.

[22] M.A. Shifman and A.I. Vainshtein, "Solution of the Anomaly Puzzle in SUSY Gauge Theories and the Wilson Operator Expansion," Nucl.Phys.B277 (1986) 456.

[23] K. Konishi and K. Shizuya, "Functional Integral Approach To Chiral Anomalies In Supersymmetric Gauge Theories," Nuovo Cim.90A (1985) 111.

[24] N. Arkani-Hamed and H. Murayama, "Renormalization Group Invariance of Exact Results in Supersymmetric Gauge Theories," hep-th/9705189; "Holomorphy, Rescaling Anomalies and Exact beta Functions in Supersymmetric Gauge Theories," hep-th/9707133.

[25] J.H. Brodie and A. Hanany, "Type IIA Superstrings, Chiral Symmetry, and N=1 4D Gauge Theory Dualities," hep-th/9704043, Nucl.Phys. B506 (1997) 157-182.

[26] K. Intriligator and N. Seiberg, Lectures on supersymmetric gauge theories and electricmagnetic duality, hep-th/9509066, Nucl.Phys.Proc.Suppl. 45BC (1996) 1.

[27] I. Affleck, M. Dine and N. Seiberg, "Dynamical Supersymmetry Breaking in Supersymmetric QCD", Nucl. Phys. B241 (1984) 493-534.

[28] K. Hori, "Consistency Conditions for Fivebrane in $M$ Theory on $\mathbf{R}^{5} / \mathbf{Z}_{2}$ orbifold", hepth/9805141.

[29] K. Landsteiner, E. Lopez and D.A. Lowe, "N=2 Supersymmetric Gauge Theories, Branes and Orientifolds", hep-th/9705199, Nucl.Phys. B507 (1997) 197-226.

[30] K. Izawa and T. Yanagida, "Dynamical Supersymmetry Breaking in Vector-like Gauge Theories", hep-th/9602180, Prog.Theor.Phys. 95 (1996) 829-830.

[31] K. Intriligator and S. Thomas, "Dynamical Supersymmetry Breaking on Quantum Moduli Spaces", hep-th/9603158, Nucl.Phys. B473 (1996) 121-142.

[32] K. Intriligator and P. Pouliot, "Exact Superpotentials, Quantum Vacua and Duality in Supersymmetric $S P\left(N_{c}\right)$ Gauge Theories", hep-th/9505006, Phys.Lett. B353 (1995) 471476. 
[33] K. Intriligator and N. Seiberg, "Duality, Monopoles, Dyons, Confinement and Oblique Confinement in Supersymmetric $S O\left(N_{c}\right)$ Gauge Theories", hep-th/9503179, Nucl. Phys. B444 (1995) 125. 